\title{
Comparison of Bus Network Structures in Face of Urban Dispersion for a Ring-Radial City
}

\section{Hugo Badia ${ }^{1}$}

Published online: 3 September 2019

(C) The Author(s) 2019

\begin{abstract}
This paper extends the discussion about the bus network design in face of the mobility patterns associated with different degrees of urban dispersion. Based on an analytical approach, a comparison of total costs among different network structures is made for a ring-radial city, which is the other most common regular city layout. The results clarify what structure is the best solution for different scenarios of dispersion and city and transport characteristics. Simultaneously, the effect of the street pattern on the applicability of each structure is evaluated comparing these results to a previous research for a grid city. Three basic structures are analyzed: a radial scheme, a direct-trip-based network, and a hybrid structure as a transfer-based alternative. Each structure is dominant for a specific range of dispersion: radial networks for scenarios of high concentration, direct-trip-based systems for intermediate degrees of dispersion, and transfer-based structures when the activities are decentralized. However, constraints on stop capacity modify these ranges. Each structure presents a different distribution of travel time in the transit chain and agency costs that determine the most competitive alternative. In addition, the behavior of the structures and the evolution of costs regarding urban decentralization are practically the same for both street patterns: system costs grow with increasing mobility dispersion, the range of applicability for each structure is the same, and changes in their applicability when main input parameters vary are very similar. Therefore, these results make it possible to generalize about the conclusions obtained independently of the street layout.
\end{abstract}

Keywords Transit network design · Bus network structure $\cdot$ Ring-radial networks $\cdot$ Hybrid network $\cdot$ Public transportation $\cdot$ Urban mobility

Hugo Badia

hubr@kth.se

1 Division of Transport Planning, KTH Royal Institute of Technology, Brinellvägen 23, SE-100 44 Stockholm, Sweden 


\section{Introduction}

A transit system is the key transport mode for urban mobility when faced with congestion, pollution, space degradation, or inefficient energy consumption derived from the excessive use of automobiles. However, the development of efficient transit systems - in order to compete with private vehicles - has to face the process of increasing urban sprawl, which generates complex mobility patterns due to changes in urban land use (Sun et al. 2007). This aspect reduces the successful implementation of this transport mode and opens a discussion about how transit networks should be designed to overcome this handicap (Dodson et al. 2011). Focusing on that discussion, this research connects the transit network design problem with the evolution of the urban form.

Some authors such as Anas et al. (1998) and Rodrigue et al. (2006) have summarized the evolution of urban forms in three basic phases. At the beginning, a city is highly centralized, with most of activities being concentrated in the central city core. This core is surrounded by residential areas, shaping a centripetal vector of displacements. In a second phase, activities are progressively relocated to areas adjacent to the initial center. The city core is expanded and new trips that do not depend on the initial center appear. Finally, the last stage is a dispersed urban form where new activities are located in external areas far from the city center. Although cities tend toward dispersion, each of them is in a particular stage of this process. Some of them retain a strong center, while at the other extreme, others are completely dispersed with no centrality. Different studies such as Bontje and Burdack (2005), Riguelle et al. (2007), and Lee (2007) show this evolution and disparity of the degree of urban sprawl in European and American cities.

There is a connection between urban form and mobility patterns (Aguilera 2005). Therefore, the previous urban sprawl process changes the initial centripetal pattern, where destinations are in the city center, to complex scenarios, where peripheral trips out of the traditional center account for a higher percentage of journeys. This evolution of the mobility pattern evinces that an initial radial scheme for transit networks cannot be a competitive alternative to satisfy the increasing number of peripheral trips. As a consequence, an alternative network design approach is needed if transit systems are to be an attractive choice among transport modes. In the literature, authors like Mees (2000) and Nielsen et al. (2005) compare two main design approaches called, in this paper, the direct-trip-based structure and the transfer-based structure, which is also named hybrid network.

In the former, the original radial network remains and additional lines are introduced to connect the new required displacements through direct services. This approach is based on the fact that planners assume that transfers are perceived negatively and therefore seek to limit their number. In this line, some design models of transit networks seek to minimize the number of transfers (Zhao 2006) or restrict the number to a certain maximum (Baaj and Mahmassami 1995). The resultant networks following this approach are diffuse systems consisting of the sum of many lines that operate independently from each other without working as a real network and have a low understandability that makes their usage difficult. For that reason, the previous authors (Mees 2000; Nielsen et al. 2005) question whether this is the best alternative, especially with the increasing mobility disparity, which emphasizes the weaknesses of this kind of structure. 
Mees (2000) and Nielsen et al. (2005) defend the transfer-based design approach in a qualitative way. The main characteristic of this structure is a simple scheme composed of a small number of lines adapted to the street pattern. This design makes the operation more efficient and users can take advantage of its high understandability. The main change with regard to the direct-trip-based design approach is the role of transfers. In the transfer-based structure, transfers are an essential step in completing most trips. The direct-trip-based structure has been the most widely used in cities, but some of these cities (Vancouver, Copenhagen, Stockholm, Barcelona, etc.) have completely or partially redesigned their bus networks using a transfer-based structure in the last decades.

Reviewing the literature on transit network design, we find recent analytical models that are suitable tools for the design of transfer-based structures. Daganzo (2010) proposed a hybrid structure composed of a central grid in the city core and a huband-spoke scheme in the periphery. By means of this model, we can define the general layout of a simple bus network that is adjusted to a grid street pattern. One example of this is Estrada et al. (2011), where the model was used for the design of a new bus network in Barcelona. On the other hand, Badia et al. (2014) extended the application of the hybrid scheme on a ring-radial street pattern, and Chen et al. (2015) compared the design of the hybrid structure for both street layouts. However, these contributions do not discuss the advantages and weaknesses of the design approach behind the hybrid network, that is, a transfer-based structure, in comparison to the direct-trip-based strategy. Therefore, a quantitative comparison is lacking.

Authors like Thompson (1977) and Newell (1979) compared alternative design approaches in extreme scenarios of complete dispersion or high concentration. The former defended transfer-based structures due to the increasing urban sprawl, while the latter considered that the most suitable solution was still a network focused on the traditional center since that center remained as the main focus of demand. Recently, more complete formulations have been developed in Badia et al. (2016) and Fielbaum et al. (2016) to answer the same question. Although they work with different methodologies, their final objective is the same: to create a tool to help make strategic decisions about the most efficient network structure depending on the evolution of the urban form.

These last two papers (Badia et al. 2016; Fielbaum et al. 2016) work with general outlines of the urban form where different degrees of demand dispersion are represented. For their simplified cities, different network structures are compared to determine the most suitable solution in a city with a specific degree of dispersion. On the one hand, Fielbaum et al. (2016) use a hierarchical description of the city by means of a graph. This description is introduced in Fielbaum et al. (2017). How the demand is distributed among the different nodes defines the level of decentralization. On the other hand, Badia et al. (2016) work with a city as a continuous area where the size of a central attractant zone, which is the zone where all the destinations are located, determines the mobility pattern for a specific degree of dispersion. With this city representation, the model includes the access cost in the comparison of network structures, which is omitted in Fielbaum et al. (2016).

Focusing on Badia et al. (2016), the design of network structures is adapted to a specific street pattern, namely a grid. This factor opens the question of what happens if that street layout changes. To answer this question, in the current paper, a comparison among network structures is made considering a circular city characterized by a ring- 
radial street pattern. This is the other regular pattern widely found in urban areas (Dickinson 1961; Lynch 1962) and studied in transit networks (Vaughan 1986; Badia et al. 2014; Chen et al. 2015; Chen and Nie 2018). In order to make this alternative comparison, we adapt the pre-existing models to describe the operation of the different structures on that ring-radial street pattern: radial, direct-trip-based and transfer-based structures. For this last network, the starting model is the ring-radial hybrid model presented in Badia et al. (2014), although the original model is modified to represent different scenarios of demand dispersion. Following the same approach, new formulations are derived for the other two structures. In this way, the formulations presented in this paper extend the applicability of this tool at the strategic level of bus system planning to most regular cities. At the same time, to determine the effects of the street layout, the new results are matched with the previous results obtained on a grid. Finally, we consider the effects on the applicability of these networks if we include a constraint on the service headway, a factor that is not examined in Badia et al. (2016).

The exposition of this paper is as follows. Section 2 presents the analytical model used for the transit network design. The results of the comparison among network structures are compiled in Section 3, where the area of applicability of each structure is identified. Then, Section 4 compares the behaviors in the two street patterns: ring-radial and grid. Finally, Section 5 summarizes the most important conclusions and Section 6 proposes future research lines.

\section{Transit Network Design Model}

In this paper, an analytical model is used for the design of the network structures compared. The advantages that justify the appropriateness of this kind of model were emphasized in Daganzo et al. (2012): fewer data requirements, reduced computational complexity, improved system representation, transparency, and insightfulness. These models are useful tools to obtain general insights about the behavior of transport systems. This approach is widely used and recently new formulations have been proposed. Moccia and Laporte (2016) make a comparison of transit modes to identify the best choice in different scenarios of fixed demand. The comparison is extended to scenarios with elastic demand in Moccia et al. (2017). Rahimi et al. (2018) propose a model for the design of demandresponsive services. Chen and Nie (2018) present an alternative feeder service to complement a network composed by fixed routes. In Fan et al. (2018), the authors propose a model for the design of bimodal transit networks. Li and Wang (2018) make a combined design of cordon tolls for private vehicles and the bus service. Other authors include the effects of different aspects on the transit network design: urban congestion (Amirgholy et al. 2017), operating strategies (Chen et al. 2018) and greenhouse gas emissions (Cheng et al. 2018). Finally, Leurent et al. (2019) propose a comprehensive model for the design of multimodal networks integrating modal choice, traffic congestion and environmental impacts. All of these contributions reinforce the applicability of analytical models for the study of transit systems.

The starting point for the model developed in this study is Badia et al. (2014), in which the ring-radial hybrid network scheme is presented. However, this is modified in order to distinguish different degrees of demand dispersion and to design alternative 
transit network structures. The next subsections define the model in detail: city and demand representations, the geometry of the network structures, the objective function and optimization, and finally, the partial costs and user transit chains.

\subsection{City and Demand Representations}

The urban area that the transit system serves is assumed to be a circular city whose size is defined by a radius $R$ and whose street pattern is a homogeneous and infinite ringradial mesh. Regarding transit demand, the model works with an average hourly demand $\lambda$ that has a maximum peak during the rush hour with a value of $\Lambda$. It is assumed that these values do not vary with the level of service or other factors related to the transit supply. The spatial distribution of demand follows the same approximation as in Badia et al. (2016); that is, all the demand is attracted to a central area but is generated over the whole city. Parameter $\phi$ determines the extension of the central attractant area, which is a circle of radius $\phi R$, as shown by Figs. 1 and 2 . In this central area, destinations are uniformly distributed. On the other hand, there are origins in the central area and in the rest of the city. They are uniformly distributed again, although with different densities in each area. The central density is higher than the peripheral one by a factor of $f_{d}$. The parameter $\phi$ varies from 0 to 1 , representing high centralization or high dispersion of mobility respectively. The parameter $f_{d}$ is 1 when the density of origins is the same in the whole city or greater when that density is higher in the central circle of radius $\phi R$. Given this distribution, trips are classified into two categories with regard to the locations of their origins: (i) trips with origins inside the central attractant area, that is, central trips whose probability is $\rho=f_{d} \phi^{2} /\left[1+\phi^{2}\left(f_{d}-1\right)\right]$, and (ii) trips with origins in the periphery, that is, centripetal trips whose probability is $(1-\rho)=\left(1-\phi^{2}\right) /\left[1+\phi^{2}\left(f_{d}-1\right)\right] .^{1}$

This spatial distribution of demand is also assumed in other papers such as Smeed (1965), Tan (1966), and more recently Tsekeris and Geroliminis (2013) and Li et al. (2014). It is simple enough to take advantage of the clarity of analytical models. Only two parameters represent different degrees of dispersion for a monocentric mobility pattern. This assumption improves the uniform distribution over the whole city for generation and attraction accepted in the previous contributions about the hybrid model (Daganzo 2010; Badia et al. 2014; Chen et al. 2015). The distribution studied of those papers does not allow different scenarios of demand dispersion to be considered.

The monocentric approach is assumed since it is the predominant urban structure. In spite of the urban sprawl process and the existence of new centers, the traditional centers remain as the predominant focus of demand. Bertaud (2004) proposes a model of the mono-polycentric city, where the central district is still the most relevant although the initial monocentrism progressively evolves to polycentrism. Similar results are

\footnotetext{
${ }^{1}$ The total number of origins is $\lambda$, some of them $\lambda_{c}$ are in the central area and the rest of them $\lambda_{p}$ in the periphery, then $\lambda=\lambda_{c}+\lambda_{p}$. According to the spatial distribution of demand, we assume that the central density $\lambda_{c} / \phi^{2} R^{2}$ is $f_{d}$ times higher than the peripheral density $\lambda_{p} /\left(1-\phi^{2}\right) R^{2}$, that is, $\lambda_{c} / \phi^{2} R^{2}=f_{d} \lambda_{p} /\left(1-\phi^{2}\right) R^{2}$. Knowing these two relationships, we can obtain the number of origins in the central area and in the periphery: $\lambda_{c}=\lambda f_{d} \phi^{2} /\left[1+\phi^{2}\left(f_{d}-1\right)\right]$ and $\lambda_{p}=\lambda\left(1-\phi^{2}\right) /\left[1+\phi^{2}\left(f_{d}-1\right)\right]$ respectively. The probability of central trips is the number of origins in the central area $\lambda_{c}$ divided by the total number of origins in the city $\lambda: \rho=\lambda_{c} / \lambda=f_{d} \phi^{2} /$ $\left[1+\phi^{2}\left(f_{d}-1\right)\right]$. The probability of centripetal trips is the number of origins in the periphery $\lambda_{p}$ divided by the total number of origins in the city $\lambda$, this is the complementary of $\rho:(1-\rho)=\lambda_{p} / \lambda=\left(1-\phi^{2}\right) /\left[1+\phi^{2}\left(f_{d}-1\right)\right]$.
} 
shown in Giuliano and Small (1991), Craig and Ng (2001), Baumont et al. (2004), Griffith and Wong (2007), and Pereira et al. (2013), reinforcing the idea that monocentrism is still predominant; that is, the clusters of high demand are located around the traditional city center. However, the deconcentration process is a general tendency since new centralities have appeared. Their densities are higher in comparison with their surroundings but not in comparison with the whole city.

For all of the above reasons, the model used in this paper considers an idealized monocentric city whose center tends to expand, following a process that gradually increases the degree of urban dispersion. Activities are initially concentrated in a small center and progressively occupy larger extensions due to the spread of that center; the parameter $\phi$ controls this process. On the other hand, the parameter $f_{d}$ balances the weights of central and peripheral areas on the mobility.

\subsection{Geometry of Transit Network Structures}

Three basic structures found in the literature (Vuchic 2005) are compared in this study: a radial network, a direct-trip-based one, and a transfer-based system represented by the hybrid scheme. Figure 1 shows the geometry of this hybrid network, which is explained in detail in Badia et al. (2014). This is composed of a ring-radial mesh (radial and circular lines) in the central part of the network and a hub and spoke scheme (only radial lines) in the external area. Four decision variables define its configuration: the angle between radial lines in the central area $\theta$, which is coincident with the angular spacing between stops of circular lines; the circular line spacing $s$, which is equal to the stop spacing in radial lines; the central area size defined by the variable $\alpha$, where $\alpha R$ is the radius of the central ring-radial mesh; and the service headway $H$ for the lines in that central area. At the same time, the decision variable $s$ fixes the branching of radial lines in the periphery. These lines branch after every $s$ units of distance in the radial direction. In this way, the angle between radial lines decreases with the distance from the city center. At a distance $r$, this angle is $\theta \alpha R / r$.

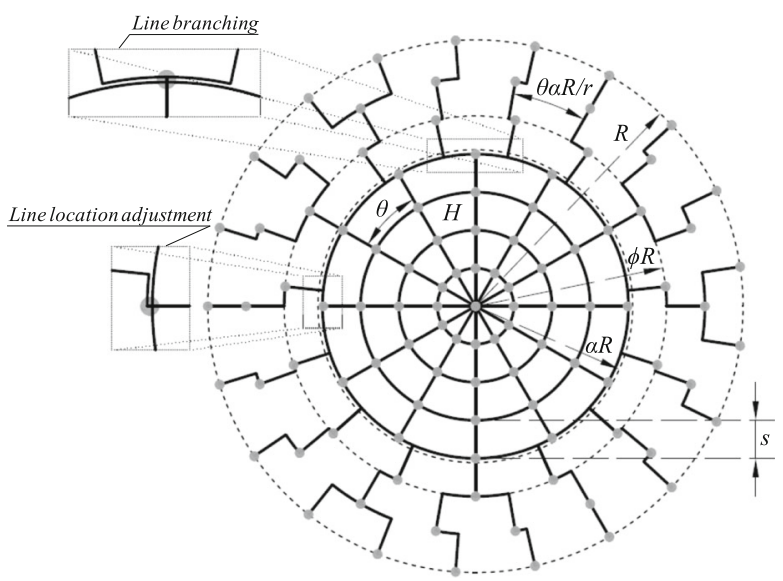

Fig. 1 Network scheme, geometric decision variables, and parameters of the ring-radial hybrid model (Badia et al. 2014) 
The central region from a supply point of view, whose area is $\pi(\alpha R)^{2}$, covers an area equal to or smaller than the central attractant region from a demand point of view, whose area is $\pi(\phi R)^{2}$, that is, $\alpha \leq \phi$. It does not make sense to have circular lines outside the attractant area since nobody would use them. When one radial and one circular line are combined, users use the most central circular line between the origin and destination because this path is always the shortest. Any trip whose origin is out of the attractant area has a more central destination because all the destinations are in that attractant area. Therefore, the circular line chosen in this case is always that one that serves the destination.

In the central area, a simple structure and service headway prevail over spatial coverage. Headway is constant in the central mesh while the stop spacing increases in the angular direction. On the other hand, spatial coverage prevails in the periphery. Line branching guarantees a constant spatial coverage equal to the boundary $\alpha R$, but headway increases progressively with distance from that boundary. The other two structures always follow this second criterion; that is, spatial coverage is constant across the whole city.

Figure 2a shows the radial scheme, which is a particular case of the hybrid structure for $\alpha=0$. Therefore, it follows the same behavior as the peripheral section of that previous structure. In this case, only radial lines serve the city and these start to branch out from its center. There are no circular lines, an important factor that differentiates this structure from a hybrid network, which has at least one circular line even for small values of $\alpha$. The decision variables are $s, \theta$, and $H$. Variables $s$ and $\theta$ define the network geometry and the branching of the radial lines that guarantees the same spatial coverage across the city. $H$ is the headway of one corridor at the central point of the network, and this headway increases with distance from that center.

At the same time, that radial network is the starting point for the direct-trip-based structure (Fig. 2b). The objective of this third structure is to satisfy all trips through direct connections without transfers. To achieve this goal, we follow the same strategy as in a grid street layout (Badia et al. 2016); that is, the central attractant area is divided into concentric circular swaths. The radial network serves the central one and additional groups of lines are gathered in the rest. Every stop is served by one line of the radial

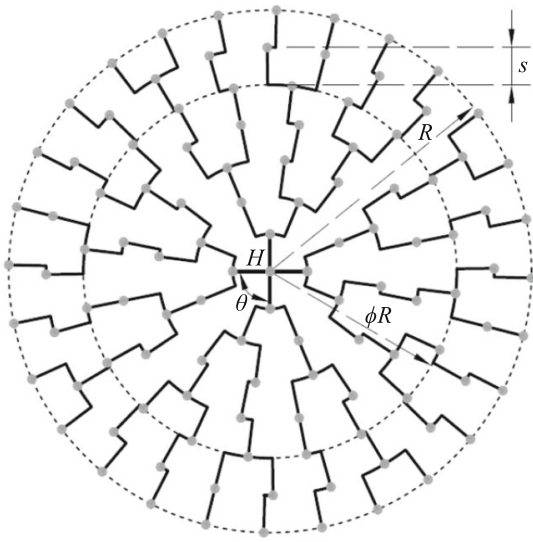

(a) Radial structure

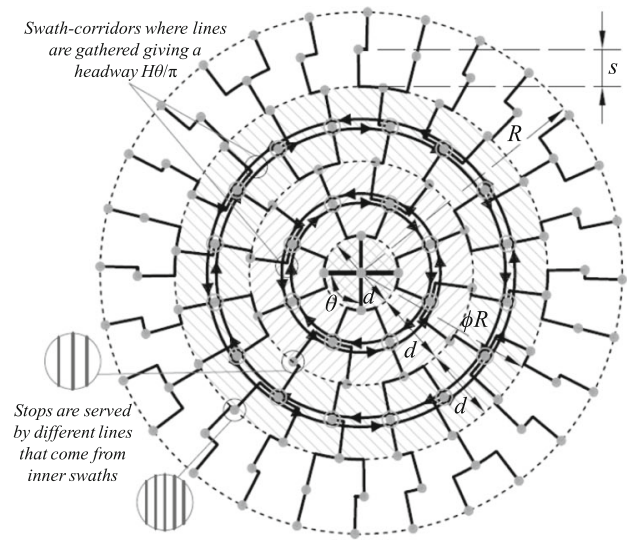

(b) Direct-trip-based structure

Fig. 2 Network scheme, decision variables, and parameters of the radial and direct-trip-based structures on a ring-radial street pattern 
network and two lines of each inner swath-corridor with regard to the own stop. As Fig. $2 \mathrm{~b}$ shows, one stop located between the first and the second swath-corridor is served by three lines: one line belongs to the radial scheme that serves the central point of the city and two lines that come from the first swath-corridor. On the other hand, if the stop is located beyond the second swath-corridor, five lines serve the stop: one line that comes from the central point, two from the first swath-corridor and other two from the second swath-corridor.

To complement the previous explanation, Fig. 3 shows the route layout of some lines that connect one swath to two external sectors. One of these lines crosses the external area between the city boundary and the connected swath following the same path as the radial network. Once the line arrives at the swath, it runs along the swathcorridor longitudinally, approximately defining a semicircle. After that, the line crosses the external area again but on the opposite side of the city. In this way, this line connects half of the swath with its zone of influence in the external area. A symmetrical line that runs over the other semicircle connects the other half of the swath to the same two external sectors. For example, Fig. 3 shows three of these pairs of lines, L1-L4, L2-L5, and L3-L6, which go into the second swath at the same point. If this process is repeated for all the lines that arrive at the swath at the remaining entrance points to the swathcorridor, this swath will be connected with the whole of the city by direct services. The entrance points are coincident with the stops in the swath-corridor. The connection with the inner area of the swath is already achieved by means of the lines that serve inner swaths.

Four decision variables determine the configuration of this structure: three from the previous radial network $(s, \theta$, and $H$ ) and an additional one that defines the swath width $d$. Focusing on the initial radial network, $H$ is the headway for each corridor in the city center and increases due to the line branching. To maintain the vehicle flow, at a distance $r$, the headway is $(r / s) H$. In the rest of the swaths, it is assumed that the same level of service is supplied from the point of view of the headway. That is, a line that serves a swath located at a distance $r$ has, on average, a headway of $(r / s) H$. In the
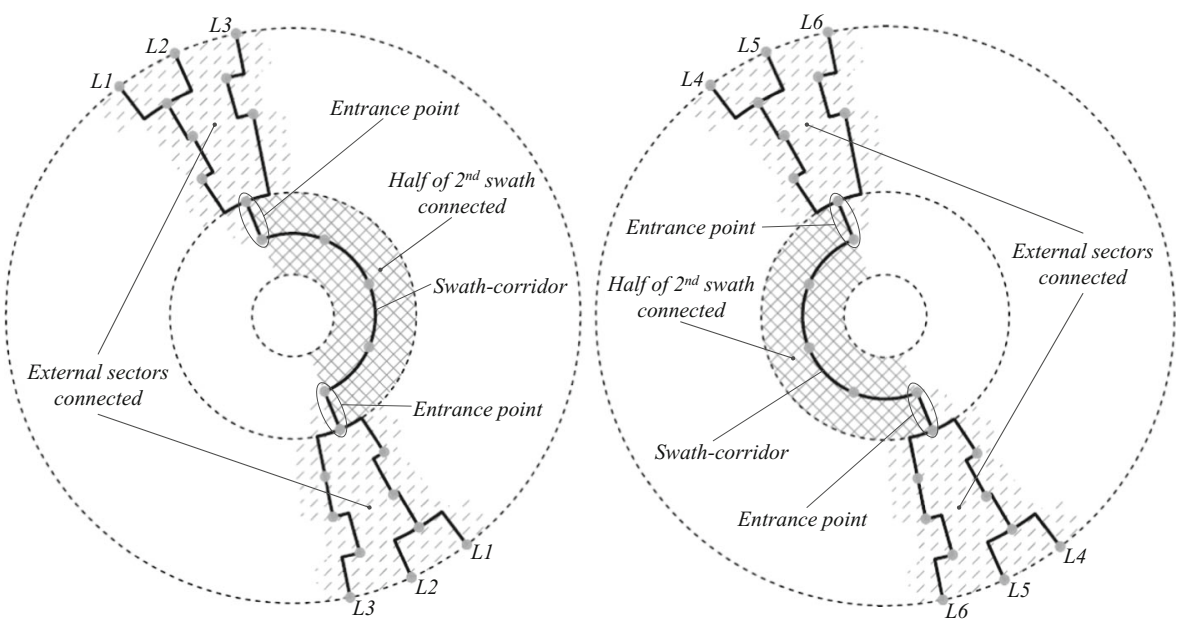

Fig. 3 Detail of a direct-trip-based network structure: how lines are arranged to connect the second swath to its external area 
swath-corridor, the headway results from the addition of the different overlapping lines. From Fig. 3, it is possible to deduce that the number of overlapping lines is $\pi / \theta_{r}$, where $\theta_{r}$ is the angle between radial lines at a distance $r$ from the city center. Knowing that $\theta_{r}=\theta s / r$, the resultant headway in each swath corridor is $H \theta / \pi$. On the other hand, the stops are served in the radial direction by different lines of the inner swath-corridors as explained above and shown in Fig. 2b. The number of overlapping lines per stop at a distance $r$ is $1+2(r-d / 2) / d$. That is one line from the center and two lines per each inner swath-corridor, being $(r-d / 2) / d$ the number of inner swath-corridors. As the headway per line is $(r / s) H$, the total headway at stops is $H d / 2 s$.

\subsection{Model Optimization}

The goal of this paper is to obtain the cheapest network structure with regard to the total system cost to serve a specific city with a certain mobility pattern. First, we need to determine the optimal network configuration for each structure, and then the cheapest one is the best alternative to serve a specific study scenario. Therefore, the model minimizes the total cost $Z$, which is the sum of the agency $\operatorname{cost} C_{A}$ and user $\operatorname{cost} C_{U}$. The objective function (1) includes the infrastructure length ${ }^{2} L$, kilometers travelled $V$, and fleet $M$ from the agency investment and the access time $A$, waiting time $W$, invehicle time $T$, and transferring time that users spend on their trips. Regarding transfers, two types of costs are considered: a waiting time included in the total waiting time $W$ and a walking distance understood to mean the transfer cost derived from the interruption of the trip (Daganzo 2010). This second component depends on the average number of transfers per trip $e_{T}$, a fixed walking distance $\delta$ per transfer and the pedestrian speed $w$. To add all these costs, agency costs are translated into units of time and prorated per user by a factor $\epsilon_{i} / \lambda \mu$. $\epsilon_{i}$ is the unit cost of each component of the agency cost (where $i=L, V, M$ ) and $\mu$ is the value of time. Finally, user costs are penalized by the respective perception factor $w_{j}$ (where $j=A, W, T, t$ ).

$$
\min \left\{Z=C_{A}+C_{U}=\left[\epsilon_{L} L+\epsilon_{V} V+\epsilon_{M} M\right] / \lambda \mu+\left[w_{A} A+w_{W} W+w_{T} T+w_{t}(\delta / w) e_{T}\right]\right\}
$$

subject to:

$$
\begin{aligned}
& s>0 ; 0<\theta \leq \pi / 2 ; H \geq H_{\min }>0 ; s / R \leq \phi ; O \leq C, \text { for a radial network } \\
& d \geq s>0 ; 0<\theta \leq \pi ; \min \{\theta / \pi ; d / 2 s\} H \geq H_{\min }>0 ; s / R \leq \phi ; d / R \leq \phi ; O \leq C, \\
& \quad \text { or a direct-trip-based network } \\
& s>0 ; 0<\theta \leq \pi ; H \geq H_{\min }>0 ; s / R \leq \alpha \leq \phi ; O \leq C, \text { for a hybrid network }
\end{aligned}
$$

The minimization is conditioned by geometrical (due to the derivation of the formulation), physical, operating (minimum service headway $H_{m i n}$ ) and vehicle capacity

\footnotetext{
2 The infrastructure cost per bus stop is neglected since this cost is small in comparison with the lane cost. This is acceptable in bus systems such as TRB (2003) emphasizes: "most bus stops are located along streets and consist of a waiting area integrated with the public sidewalk, signage to mark the bus stop, and, in some cases, a bench or small shelter". However, this cost would be relevant for tram or metro systems. In these other cases, the construction of stations is expensive especially when the station is underground. To add this cost, we should multiply the unit cost per station by the number of stations in the network. The number of stations is the total infrastructure length $L$ divided by the infrastructure length per stop $l_{s}$, which are defined in Table 1 . This value is considering unidirectional stations, that is, we assume a different station per direction.
} 
(vehicle occupancy $O$ lower than capacity $C$ ) constraints (2). Regarding the minimum headway constraint, we check the most congested stops of the network. For the radial scheme, these stops are at the central point where the headway per direction is $H$. For the hybrid network, the most congested section is each central corridor whose headway is $H$. In the direct-trip-based structure, the greatest flow of buses is on the swathcorridors in the circular direction, where the headway is $H \theta / \pi$, or at any stop in the radial direction, whose headway is $H d / 2 s$.

The objective function for all network structures is convex, as can be seen in Appendix 3 and in detail in Badia (2016), which include a sensitivity analysis of this model; that is, the local optimum found is the global solution. This characteristic and the small number of decision variables make its minimization trivial. Therefore, its optimization is performed easily by means of a grid search. The optimal network configuration is defined from the combination of values of the decision variables that gives the lowest total cost. However, this solution is only accepted under the condition that it meets all the constraints. If the global optimum breaches some of them, the final configuration will be the cheapest among the feasible range of alternatives with regard to those constraints.

\subsection{Partial Costs Formulation}

The formulation of the different agency and user costs included in the objective function is shown in this section. In addition, Appendix 1 includes a detailed explanation of the derivations of the partial costs. All of them are expressed in a compact form as a function of the decision variables.

\subsubsection{Agency Costs}

Table 1 summarizes the agency partial costs. The network structure geometry and service headway are the factors that determine these costs. The same table also includes other relevant network properties such as the average commercial speed of transit vehicles $v_{c}$ and the average infrastructure length per stop $l_{s}$.

\subsubsection{User Costs}

The paths followed by users determine the estimations of partial user costs. The same criteria for route choice as in Badia et al. (2016) are assumed in this case; a user generally chooses: (i) the closest stop to his or her origin and destination, (ii) the shortest-distance path, and (iii) the path with the minimum number of transfers. Although all these criteria are coincident in most of the trips, this is not the case in a minority of them. In these last trips, it is assumed that one criterion prevails over the others, depending on the main goal of each network structure. The purpose is to obtain a simple formulation without a comparison among different possible alternative routes in every possible network configuration. Therefore, the routing strategy of users is simplified. In a transfer-based network, the criterion that prevails is the shortestdistance path versus the minimum number of transfers, as discussed by Badia et al. (2014) and also assumed by Chen et al. (2015). However, this discrepancy only exists when $\alpha$ and $\phi$ are not coincident. If $\alpha$ is equal to $\phi$, there is always a route that 


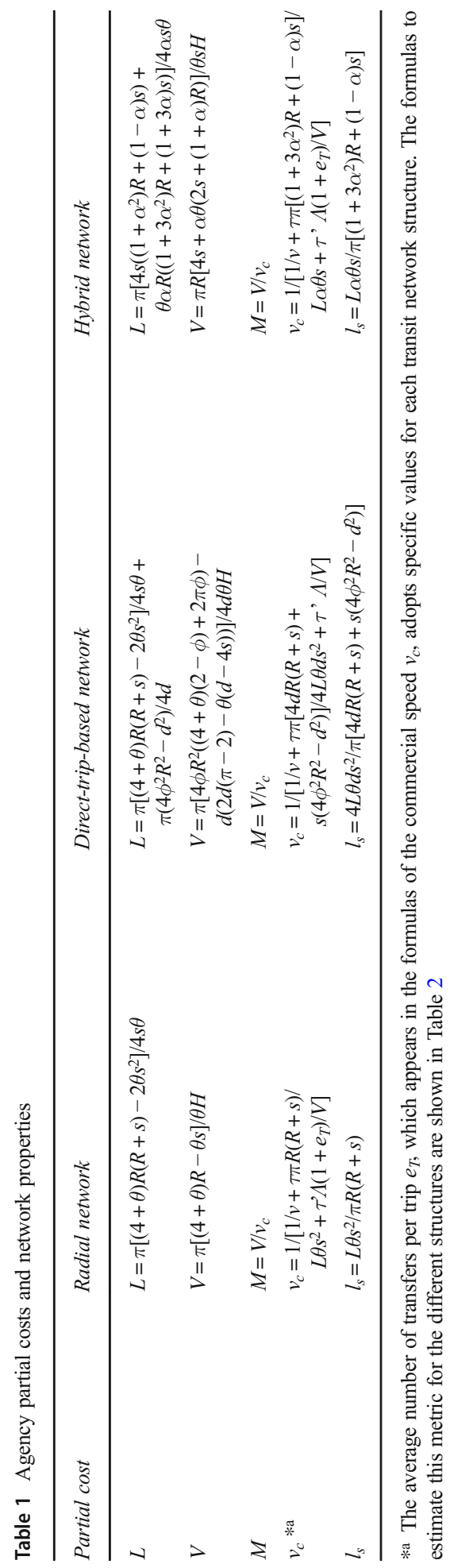


accomplishes all the criteria for all trips. In a direct-trip-based network, the criterion that prevails is the minimum numbers of transfers; that is, users always choose the direct trip. In this structure, this criterion is the best choice for users since direct trips are the alternative with the shortest total travel time. Therefore, users take advantage in the best possible way of the service that this structure supplies. A comparison between these alternative criteria is included in Appendix 2.

The preferential criterion in each case is also in line with the user behavior in real networks. Currie and Loader (2010) explore the relationship between network design and bus transfer behavior through an analysis of the Melbourne transit system. They conclude that transfer-based structures promote the combination of lines due to lower transfer penalties. These lower values are a consequence of the characteristics of this design approach: simple routes with high understandability and high frequencies. Badia et al. (2017) compare the percentage of transfer trips between the old bus network and the new redesign in Barcelona (Spain). The former was a typical direct-trip-based structure where users made transfers during only $11 \%$ of trips. The latter is a transferbased scheme that is currently in the process of gradual implementation. In this new design, that percentage reached $26 \%$ when only half of the new network was implemented. It is predicted that during the final implementation stage, $44 \%$ of trips will involve transfers. All these results suggest that the design approach conditions the user's path choice.

Figure 4 shows the different types of trips that we can find in each network structure. In the radial network, all the criteria are coincident since there is only one path that connects any origin-destination pair. This trip can be direct $\left(O_{1}-D_{1}\right)$ or can include one transfer made in the city center $\left(\mathrm{O}_{2}-D_{2,1}\right)$. In the latter case, this network presents circuitous trips whose lengths are considerably longer than the real distance between origin and destination, for example the case of $\mathrm{O}_{2}-D_{2,2}$.

In a direct-trip-based network, the trip $\mathrm{O}_{3}-D_{3}$ is an example of most of the trips where the criteria of minimum number of transfers and shortest-distance path are coincident. However, when the origin and destination are separated by an angle of at least $2+\theta / 2 \mathrm{rad}$, the shortest-distance path goes through the city center, but the path with fewer transfers goes through the respective swath-corridor. As mentioned above, users are supposed to choose this second option, that is, trip $\mathrm{O}_{4}-D_{4}$. Finally, users get off the bus at stops located along the swath-corridor, although this decision implies a longer egress time. To reach the closest stop to the destination, the trip would imply a transfer.

On the other hand, in the hybrid network, the different criteria are not coincident on the same path if trips have two characteristics: the origin and destination belong to the branched section of the radial lines and the angular distance between them is smaller than $2 \mathrm{rad}$. In this case, the prevailing criterion is the shortest-distance path; the trip $\mathrm{O}_{5}-\mathrm{D}_{5}$ in Fig. $4 \mathrm{c}$ is one instance of this. As two transfers are required during these trips, they are composed of three sections: from the origin to the first transfer point located at the most external circular line, from that point to the second transfer stop by means of that circular line, and from that last stop to the destination using a radial line. However, the first condition required can only be fulfilled when $\alpha$ is smaller than $\phi$. This kind of trips does not exist if $\alpha$ is equal to $\phi$. For the remainder of the trips, the route choice criteria are coincident. If the angular distance is smaller than $2 \mathrm{rad}$ and at least one of the extremes is located in the central ring-radial mesh, 


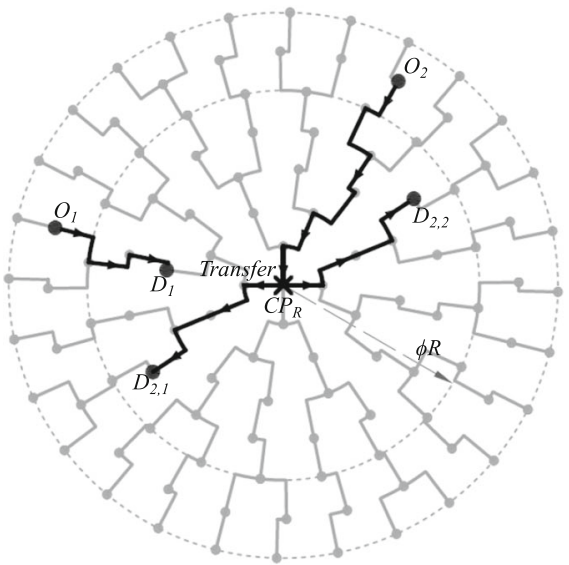

(a) Radial structure

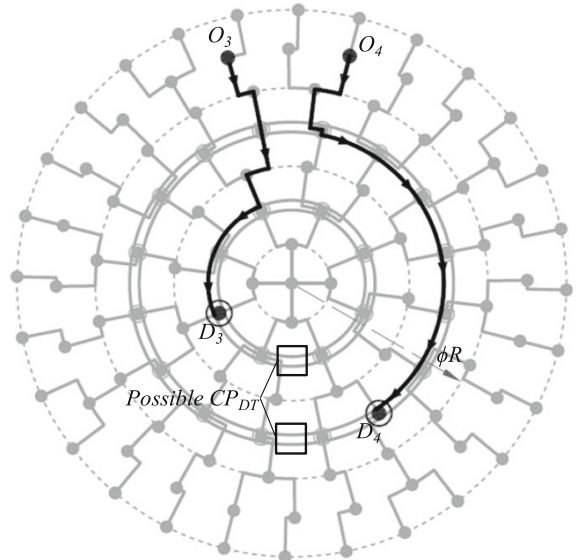

(b) Direct-trip-based structure

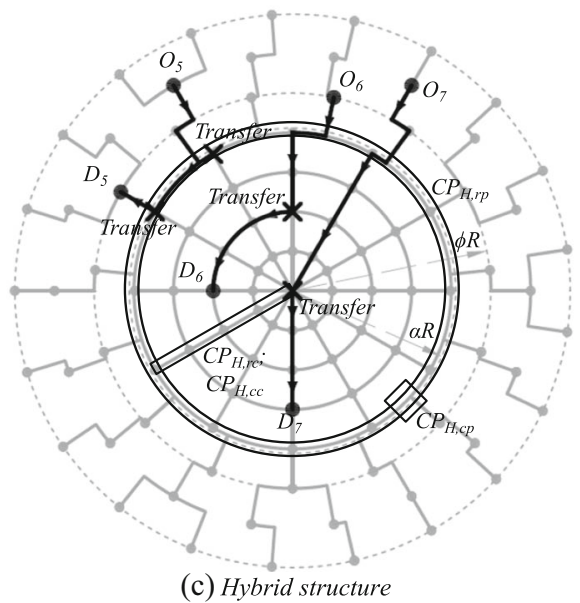

Fig. 4 Paths and critical loaded points of the structures studied on a ring-radial street pattern

the trip combines one radial and one circular line (trip $O_{6}-D_{6}$ ). However, when that angular distance is greater, regardless of the locations of the origin and destination, the trip only uses radial lines passing through the city center, where users make the single transfer required (trip $O_{7}-D_{7}$ ).

With regard to waiting costs, the system could operate according to headways or schedules. However, it is assumed that operating according to schedules is only possible in direct connections. Therefore, radial and transfer-based structures always operate according to headways in the whole city. On the other hand, direct-trip-based networks can operate in a mixed way. In a central area, where the service headways are lower than a threshold $H_{s}$, the system operates according to headways, and in the rest of the city, it operates according to schedules. An additional parameter $\varepsilon_{H}$ defines that central area as a circle of radius $\varepsilon_{H} R$. The average waiting time in this network structure depends on the relationship between $\varepsilon_{H}$ and $\phi$. Equation (3) computes the waiting time according to a service headway $H_{l}$ as used in Tirachini et al. (2010) and Badia et al. (2016). Operating according to 
headways implies that users arrive randomly at a stop, and on average, the waiting time is half of the headway. On the contrary, users arrive some minutes $h_{s}$ before the bus's arrival, that is, the safety waiting time to avoid the loss of the bus, and an opportunity cost is added to penalize the worse level of service. This last cost is proportional to the service headway by means of a factor $f_{s}$, which is called home waiting time factor and its value is smaller than 0.5 since waiting at home/work is less negative than waiting at stop.

$$
w_{s}=\left\{\begin{aligned}
H_{l} / 2, & \text { if } H_{l}<H_{s} \\
h_{s}+f_{s} H_{l}, & \text { if } H_{l} \geq H_{s}
\end{aligned}\right.
$$

Based on the previous assumptions and the resultant chosen paths, we are able to estimate the formulation of the user costs, as summarized in Table 2.

\subsubsection{Vehicle occupancy}

Finally, we estimate one property of the network, its highest occupancy, as a result of the comparison of the most loaded points. Figure 4 shows the locations of these points in the different structures. The maximum load is at the central point in a radial scheme $\left(C P_{R}\right)$. For a direct-trip-based network, the critical point belongs to one swath, which can be the most external one when the attractant area is small or an intermediate swath when this area is larger. In the hybrid network, two types of critical points are identified on the radial lines and two on the circular lines. In both cases, one critical point is located at the central ring-radial mesh boundary: $C P_{H, r p}$ for the radial lines and $C P_{H, c p}$ for the most external circular line. Depending on the size of that mesh, the other two critical points are located in the middle position of a radial line $\left(C P_{H, r c}\right)$ and on an intermediate circular line $\left(C P_{H, c c}\right)$. Table 3 includes these occupancies.

\section{Comparison of Network Structures}

First of all, we define the input parameters of the analytical model for the base case study in which we make the comparison. Table 4 summarizes all these parameters, which are the same as in Badia et al. (2016). The transit technology considered is a highperformance bus with a homogeneous fleet composed of standard buses only or articulated buses only. Mixed fleets are not accepted. Moreover, due to an irregular allocation of passengers among the vehicles, a vehicle occupancy safety factor $(S F)$ is considered.

Then, an exhaustive analysis is carried out to find out how the dispersion affects the performance of the different transit network structures and how the characteristics of the city, demand, or transport technology change their ranges of applicability. This analysis considers two different situations with regard to the headway constraint: (i) unconstrained case where $H>0$, and (ii) constrained case where $H_{\text {min }}=0.5 \mathrm{~min}$. In the former, we assume that operating measures satisfy any requirement on stop capacity, while in the latter the stops have limitations to serve great flows of buses. In this second case, the stop capacity is 120 buses/h. 


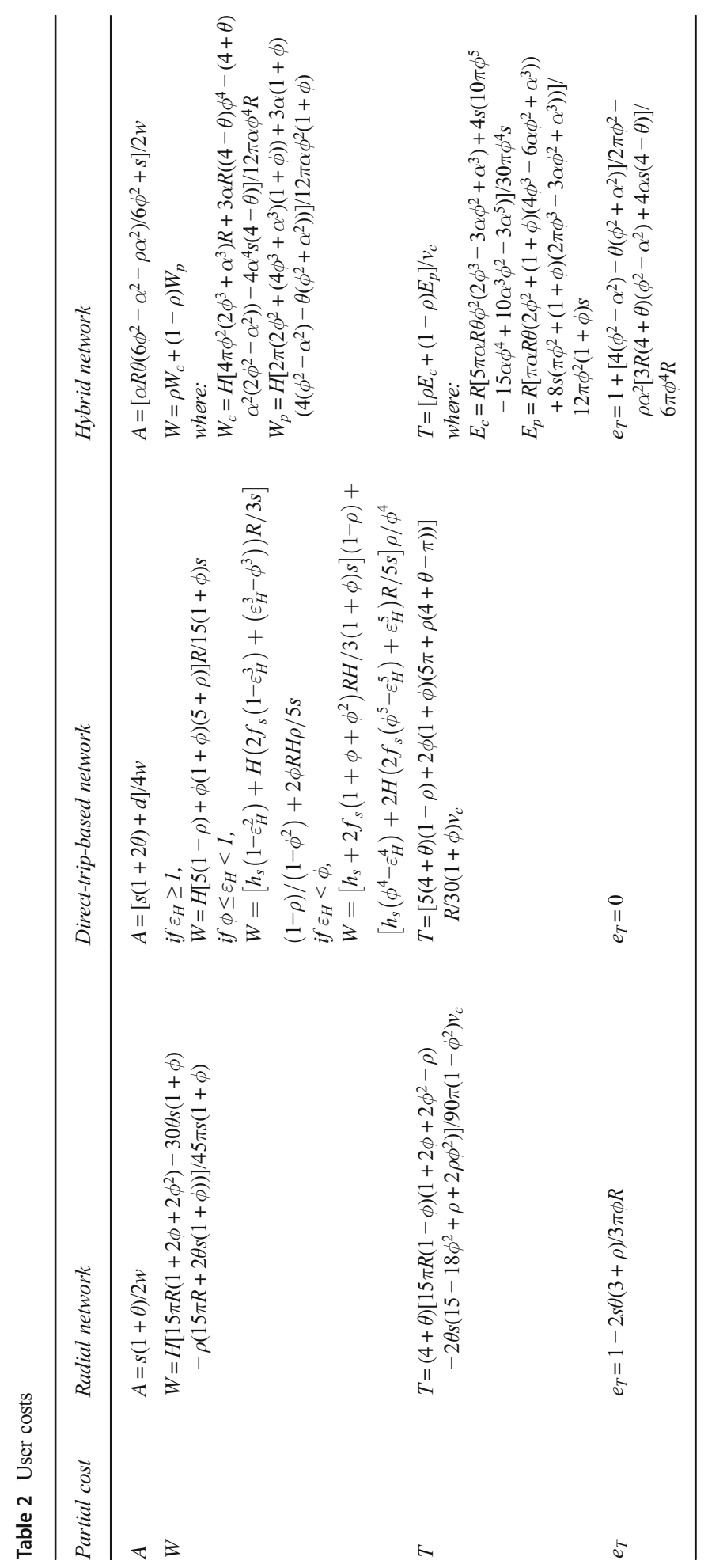


Table 3 Maximum occupancies

\begin{tabular}{ll}
\hline Network structure & Occupancy $O$ \\
\hline Radial network & $O=(S F) H \Lambda \theta[3 \pi \phi R-\theta s(3+\rho)] / 6 \pi^{2} \phi R$ \\
$\begin{array}{l}\text { Direct-trip-based } \\
\text { network }\end{array}$ & if $\left(2 d \rho+\sqrt{12 \phi^{2} R^{2} \rho+d^{2} \rho^{2}}\right) / 6 \rho \leq \phi R$, \\
& $O=(S F) H \Lambda \theta d\left[d \rho\left(36 \phi^{2} R^{2}-\rho d^{2}\right)+\rho^{1 / 2}\left(12 \phi^{2} R^{2}+\rho d^{2}\right)^{3 / 2}\right] / 216 \pi \phi^{4} R^{4} \rho$ \\
& if $\left(2 d \rho+\sqrt{12 \phi^{2} R^{2} \rho+d^{2} \rho^{2}}\right) / 6 \rho>\phi R$, \\
& $O=(S F) H \Lambda \theta d(2 \phi R-d)\left[\phi^{2} R^{2}(1-\rho)+\rho d(2 \phi R-d)\right] / 4 \pi \phi^{4} R^{4}$ \\
Hybrid network & $O=(S F) \max \left\{H \theta \Lambda\left(1-\rho \alpha^{2} / \phi^{2}\right) / \pi ; H \Lambda\left[4 R^{2}\left(\phi^{2}-\rho \alpha^{2}\right)+\rho s(4 \alpha R-s)\right]\left[4 R^{2}\left(\phi^{2}-\alpha^{2}\right)\right.\right.$ \\
& $+s(4 \alpha R-s)] / 32 \pi^{2} \phi^{4} R^{4} ; H \theta \Lambda\left[4 \phi^{2}+\rho\left(\pi \phi^{2}-2 \phi^{2}-2 \alpha^{2}\right)\right]^{2} / 16 \pi^{2} \phi^{4} \rho$, if $R$ \\
& $\sqrt{\rho\left(4 \phi^{2}+2 \rho \phi^{2}-\pi \rho \phi^{2}-2 \rho \alpha^{2}\right)} / 2 \rho<\alpha R ; H \Lambda s\left(2 \rho s+\sqrt{\rho\left(12 \phi^{2} R^{2}+\rho s^{2}\right)}\right)$ \\
& $\left(12 \phi^{2} R^{2}+s \sqrt{\rho\left(12 \phi^{2} R^{2}+\rho s^{2}\right)}-\rho s^{2}\right) / 108 \pi^{2} \phi^{4} R^{4} \rho$, if s/3+ \\
& $\sqrt{\left.12 \rho \phi^{2} R^{2}+\rho^{2} s^{2} / 6 \rho<\alpha R\right\}}$ \\
&
\end{tabular}

\subsection{Effects of Urban Dispersion on Transit Network Structures}

This analysis is divided into two parts. In the first one, only the parameter $\phi$ defines the degree of dispersion. The value of $f_{d}$ is 1 and is constant; that is, the generated demand is always uniformly distributed over the whole city. Then, we introduce the parameter $f_{d}$ in the discussion to evaluate what happens if central trips represent a greater percentage of journeys.

\subsubsection{Parameter $\phi$. Uniform Density of Generated Demand Over the Whole City}

Figure 5 shows the evolution of costs and vehicle occupancy for the three network structures: the radial network $(R a)$, the direct-trip-based network $(D T)$, and the hybrid scheme $(H y)$ as a transfer-based structure. The behavior of the structures compared shows that the most competitive network varies in dependence on the dispersion parameter $\phi$. As Fig. 5a reveals, there are cut-off points in the total system cost curves of these structures. If we focus on the unconstrained headway case, the transition is the same as in the case of the grid street pattern: a radial scheme for concentrated demands, direct trips in intermediate scenarios, and a hybrid structure when the decentralization is higher. The intersection between the curves of the two first structures occurs when $\phi=0.11$, that is, when the central attractant area is less than $2 \%$ of the city. The hybrid scheme offers the best solution when $\phi \geq 0.60$, that is, when the central attractant area covers an extension larger than $36 \%$ of the city. However, the direct-trip-based network reduces its range of applicability in favor of the other two structures when operating limitations at stops constrain the optimal headway. If $H_{\min }=0.5$, its total cost curve intersects with the total cost curves of the radial scheme and the hybrid network when $\phi$ is 0.15 and 0.58 respectively. The direct-trip-based structure undergoes the most important changes due to the overlapping lines in the swath-corridors. 
Table 4 Characteristics of the base case study

\begin{tabular}{llll}
\hline Input parameter & Variable & Units & Value \\
\hline Demand during rush hour & $\Lambda$ & $\mathrm{p} / \mathrm{hh}$ & 50,000 \\
Average hourly demand & $\lambda$ & $\mathrm{p} / \mathrm{h}$ & 20,000 \\
Radius of circular city & $R$ & $\mathrm{~km}$ & 4 \\
Value of time & $\mu$ & $€ / \mathrm{h}$ & 15 \\
Equivalent penalty distance per transfer & $\delta$ & $\mathrm{km}$ & 0.3 \\
Cruising speed & $v$ & $\mathrm{~km} / \mathrm{h}$ & 30 \\
Walking speed & $w$ & $\mathrm{~km} / \mathrm{h}$ & 4.5 \\
Unit infrastructure cost & $\epsilon_{L}$ & $€ / \mathrm{km}-\mathrm{h}$ & 76 \\
Unit distance cost & $\epsilon_{V}$ & $€ / \mathrm{veh}-\mathrm{km}$ & 0.85 or $1.1^{* \mathrm{a}}$ \\
Unit vehicle cost & $\epsilon_{M}$ & $€ / \mathrm{veh}-\mathrm{h}$ & 35 or $36^{* \mathrm{a}}$ \\
Vehicle capacity & $C$ & $\mathrm{p} / \mathrm{veh}$ & 80 or $150^{* \mathrm{a}}$ \\
Occupancy safety factor & $S F$ & - & 1.2 \\
Dwell time & $\tau$ & $\mathrm{s}$ & 35 \\
Boarding (and alighting) time & $\tau^{\prime}$ & $\mathrm{s}$ & 3 \\
Time perception weight of access & $w_{A}$ & - & 2.2 \\
Time perception weight of waiting & $w_{W}$ & - & 2.1 \\
Time perception weight of travelling & $w_{T}$ & - & 1.0 \\
Time perception weight of transferring & $w_{t}$ & - & 2.5 \\
Minimum service headway constraint & $H_{\text {min }}$ & $\mathrm{min}$ & - or $0.5^{* \mathrm{~b}}$ \\
Cut-off headway between types of services & $H_{S}$ & $\mathrm{~min}$ & 12 \\
Safety waiting time & $h_{s}$ & $\mathrm{~min}$ & 5 \\
Home waiting time factor & $f_{s}$ & - & $1 / 12$ \\
\hline
\end{tabular}

*a The first value corresponds to a standard bus and the second to an articulated bus

*b There is no minimum headway in an unconstrained situation

The reasons for these evolutions are related to how structures perform in each urban dispersion scenario. Although a radial network is a cheap solution from the point of view of the agency, the dispersion generates longer length trips and a greater number of transfers for users. On the other hand, to satisfy mobility needs by direct trips, the system counteracts the higher investment required through the loss of spatial coverage and frequency. The alternative of a hybrid network has a large number of transfers; however, this allows the system to maintain a more balanced time distribution among the different steps of the user transit chain. Shorter access and waiting times compensate for the additional transfer time.

It is important to clarify that the total cost of the hybrid scheme does not tend to the total cost of the radial network when the demand is concentrated, although $\alpha$ tends to small values for high degrees of concentration. As explained in Section 2.2, these two structures never coincide. The reason is that the hybrid scheme has at least one circular line, but this type of line does not exist in a radial network. This fact changes the estimation of some agency and user costs.

Agency and user costs show similar tendencies to the total cost, although break points appear in their curves due to constraints on capacity or spacing variables and the 


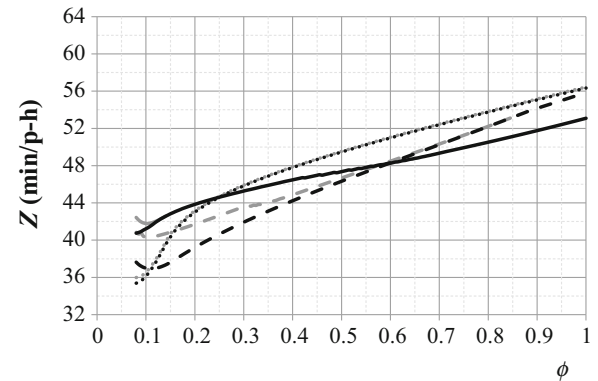

(a) Total cost

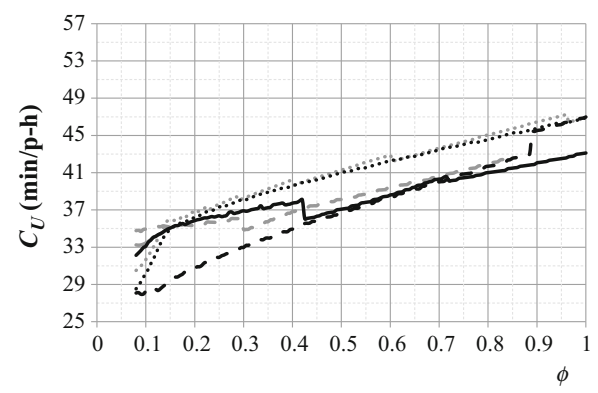

(c) User cost

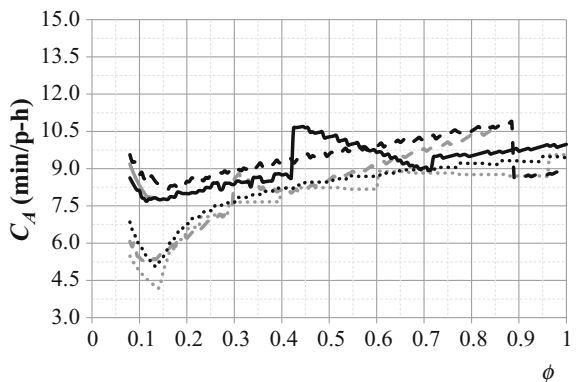

(b) Agency cost

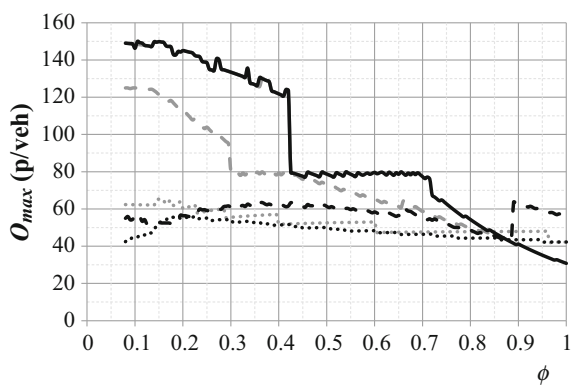

(d) Vehicle occupancy

\begin{tabular}{|c|c|c|}
\hline $\begin{array}{l}\cdots \cdot \cdot R a \text { (tmconst. } H) \\
\cdots \cdots \cdot R a \text { (const. } H \geq 0.5 \mathrm{~min})\end{array}$ & $\begin{array}{l}--D T \text { (umconst. H) } \\
--D T \text { (const. } H \geq 0.5 \mathrm{~min} \text { ) }\end{array}$ & $\begin{array}{l}-H y \text { (inconst. } H \text { ) } \\
-H y \text { (const. } H \geq 0.5 \mathrm{~min} \text { ) }\end{array}$ \\
\hline
\end{tabular}

Fig. 5 Evolution of total, agency, and user costs and vehicle occupancy with regard to the parameter $\phi$

type of service operation, which change the tendencies of the curves. First, the vehicle occupancy has an impact on the hybrid scheme. Figure $5 \mathrm{~d}$ shows high occupancies up to $\phi=0.43$, which force the system to operate with articulated buses. From that degree of dispersion up to $\phi=0.70$, the system operates with standard buses but at capacity, preventing the hybrid network from reaching a better configuration. The same capacity problems happen for the direct-trip-based structure when there is a constraint on the minimum headway. In that situation, this structure operates with articulated buses for $\phi$ $<0.3$, and with standard buses at capacity for $0.3<\phi<0.44$. Secondly, under high demand centralization, the spacing variables are constrained in the three structures due to the relation between spacings and the radius of the central attractant area $(s, d \leq \phi R)$. For low values of $\phi$, Fig. $6 \mathrm{c}$ shows the shorter spacings, which lead to greater agency costs and a reduction of user costs. Thirdly, the last break point occurs in the direct-tripbased structure at $\phi=0.89$ when the service operation changes from headways to schedules as Fig. 6a shows, with a jump in the value of the service headway. ${ }^{3}$

Figure 6 shows the evolution of the decision variables with regard to urban dispersion. First we describe this evolution when headways are unconstrained. For

\footnotetext{
${ }^{3}$ The small jumps in the curves in Figure 5 (except for Figure 5a, showing the total cost) and Figure 6 are a consequence of the optimization process. In the grid search, the different values of decision variables tested cover a large range, where each variable is varied with a specific step size. In this study, these step sizes are $\Delta H=0.05 \mathrm{~min}, \Delta \theta=2^{\circ}, \Delta s=\Delta d=0.02 \mathrm{~km}$, and $\Delta \alpha=0.005$. They are sufficiently small with regard to their applicability in real cities due to geometrical and operating constraints. Smaller step sizes would reduce these small jumps but longer computational times would be required. However, this change would not yield different results and the conclusions would be the same.
} 


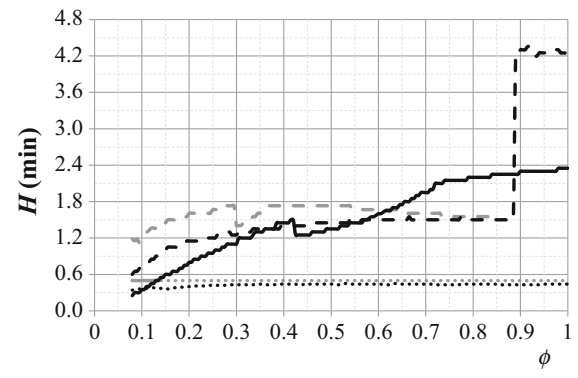

(a) Headway

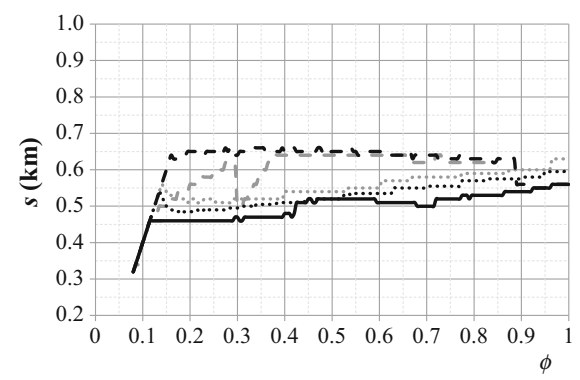

(c) Stop and line spacing

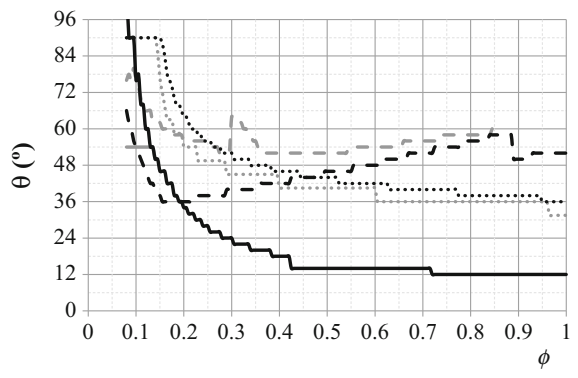

(b) Angular line spacing

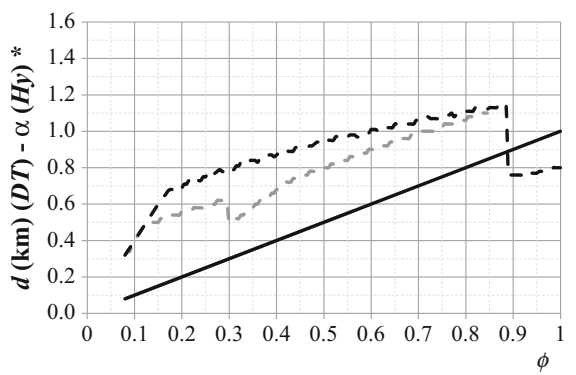

(d) Corridor spacing (DT) and decision variable $\alpha(H y)$

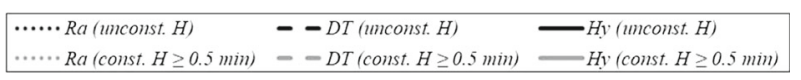

Fig. 6 Evolution of decision variables with regard to the parameter $\phi$

high levels of concentration, the spacing in the radial direction $s$ (and the swath-corridor spacing $d$ in a direct-trip-based structure) is too short, which necessitates the use of greater angular spacings $\theta$ to compensate for the growth in agency costs. Once this constraint is overcome, variable $s$ grows progressively in the radial and hybrid networks to reduce the branching of radial lines that scatters the fleet while the angular spacing decreases to guarantee the spatial coverage. On the other hand, these variables show the opposite behavior for direct trips. In this last structure, the variable $s$ is almost constant as the angular spacing increases. In order to satisfy all the trips using direct connections, the area of influence of the bus lines becomes progressively wider. That is, the greater the number of swaths, the smaller the number of lines per swath. At the same time, the value of $d$ grows to reduce the swath density for larger attractant areas.

Other important aspect of the direct-trip-based structure is the change in the type of service operation from headways to schedules. This increases the spatial coverage and the number of lines at the expense of higher headways. Besides this sudden change in the direct-trip-based network configuration, the most relevant variation of headway happens in the hybrid network, where its value grows with a more pronounced slope in scenarios under capacity constraint. The last comment is related to the size of the central ring-radial mesh of the hybrid scheme. This is completely extended over the central attractant area; that is, variable $\alpha$ is always coincident with the parameter $\phi$.

Finally, in case that there is a constraint on the minimum possible headway, the radial and hybrid networks distribute the buses over more lines, which have higher headways. Figure $6 \mathrm{c}$ shows the reduction in the angular line spacing. Additionally, we observe longer stop spacings for the radial scheme to avoid line 
branching and the associated increase of service headway with the distance from the center. On the other hand, the direct-trip-based structure increases the angular spacing to reduce the number of lines per swath-corridor; and at the same time, the distance between those corridors is reduced to increase their number for a better demand distribution.

\subsubsection{Parameter $f_{d}$. Different Generated Demand Densities Between the Central and Peripheral Areas}

Figure 7 shows what happens if the analysis includes the parameter $f_{d}$, which varies from 1 to 30 (i.e., the central density of origins is the same as the peripheral density or thirty times higher). The results reveal, just as in the grid street pattern scenario, that the parameter $\phi$ is more decisive than the parameter $f_{d}$ for determining which network structure is the best solution. We are able to identify the most competitive structure knowing only the parameter $\phi$ in practically all the cases. However, there is a certain variation in the value of $\phi$ that justify the change of structure between the direct-tripbased network and the hybrid scheme, especially for low values of $f_{d}$. This dispersion degree of change of structure decreases by $30 \%(\Delta \phi=-0.181)$ from the scenario of uniform generated demand $\left(f_{d}=1\right)$ to the analyzed scenario where the central area has the greatest weight in the mobility $\left(f_{d}=30\right)$. Capacity constraints are the reason for that variation. For low degrees of dispersion, the critical point of the hybrid network is on the radial lines at the boundary of the ring-radial mesh. All the demand generated in the periphery crosses this point. However, when the generated demand tends to be concentrated in the center, that critical point moves to an inner location of that mesh. The demand is distributed less well among the lines in the periphery than in the mesh due to the line branching. For that reason, the greater the peripheral demand, the higher the maximum occupancy. Therefore, the hybrid network configuration is further from the non-constrained optimum solution. In addition, the circular lines participate more in the central trips than in the peripheral ones; as a consequence, fewer users take radial lines when a higher percentage of generated demand is central. The other significant boundary, between radial and direct-trip-based networks, grows by only $11.8 \%(\Delta \phi=0.013)$.

A comparison of the total system costs of the different structures under the scenarios included in Fig. 7 shows the superiority of one structure in those scenarios where it is the dominant alternative over the other two strategies. In case of unconstrained headway, the radial network dominates for values of $\phi$ smaller than 0.1 , achieving a design $10 \%$ cheaper than the direct-trip-based

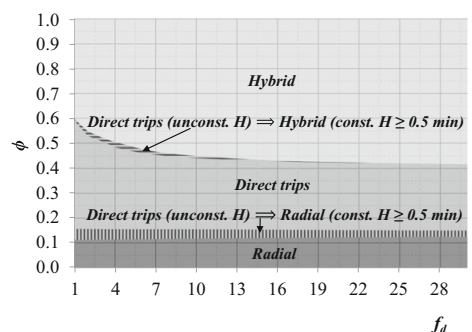

$=H y \equiv D T$ (unconst. $H) \Rightarrow H y$ (const. $H \geq 0.5 \mathrm{~min}) \quad \approx D T \quad \| D T$ (zmconst. $H) \Rightarrow R a($ const. $H \geq 0.5 \mathrm{~min}) \quad=R a$

Fig. 7 Area of applicability for the different network structures with regard to the parameters $\phi$ and $f_{d}$ 
structure and $13-14 \%$ cheaper than the hybrid scheme. Direct trips present the maximum difference compared to the radial network for values of $\phi=0.2-0.4$, where this alternative achieves a reduction of the total system cost of around 8 $10 \%$. In comparison with the hybrid structure, the most significant savings are between 7 and $12 \%$ for scenarios of dispersion $\left[\phi, f_{d}\right]=[0.15-0.25,<7]$. Finally, the hybrid network is more competitive than a radial scheme in scenarios $[\phi$, $\left.f_{d}\right]=[0.4-0.6,>10]$, being $8-9 \%$ cheaper. Compared to the direct-trip-based structure, the hybrid network shows a maximum decrease in cost of 5-6\% for high degrees of dispersion, that is, $\phi>0.85$. In short, the degree of dominance of one structure over the others varies from 5 to $14 \%$ of the total system cost reduction in this base study scenario.

In case that we introduce a constraint on the minimum headway, the boundary between the direct-trip-based structure and the hybrid network practically remains unchanged. However, the applicability of the radial network grows around $\Delta \phi=$ 0.024-0.044; the effects of the headway constraint on the direct-trip-based structure are more significant for high degrees of demand concentration (i.e., low values of $\phi$ ).

\subsection{Effects of Input Parameters on the Area of Applicability of Network Structures}

This section studies the changes in the areas of applicability for the different structures when the main input parameters of the model, namely the demand (where $\Lambda / \lambda$ is always 2.5 ), the city size, symbolized by the circular dimension $R$, the transfer penalty, and the unit costs, vary from the above base scenario. The work only focuses on the most relevant dispersion parameter $\phi$. Figure 8 exhibits the evolution of each area of applicability with regard to input parameters, which are independently analyzed with regard to the others.

In summary, three areas of applicability are identified, the same as in Section 3.1: (i) a high degree of concentration, where a radial network is the most reasonable solution, (ii) intermediate levels of dispersion, where direct connections satisfy the mobility requirements in the best possible way, and (iii) high levels of dispersion, where the most efficient alternative is a transfer-based network. However, these areas are not constant; they evolve with the variation of the input parameters. The radial scheme gains applicability in small cities and under conditions of low levels of demand, high agency costs, or low transfer penalties. The usage of direct services is promoted in small cities, at high levels of demand (or too low levels working according to schedules) and costly transfers. In opposite scenarios, the applicability of the hybrid structure increases.

However, the previous areas of applicability change if bus stops have a maximum capacity of vehicles per hour. These variations confirm that the direct-trip-based network is the most sensitive, losing competitiveness in comparison with the other two strategies of design. The negative effects on the performance of the direct-trip-based structure are more pronounced for high levels of demand. For values of $\Lambda>90,000 \mathrm{p} / \mathrm{rh}$, this structure is replaced by the hybrid scheme even for scenarios of certain centralization, where there is a high number of destinations concentrated in a small central area.

To evaluate the difference of costs between the structures compared, we focus on the unconstrained case with regard to the minimum service headway. In scenarios of high concentration, the radial network reduces the total system cost by $8-10 \%$ at most compared to the direct-trip-based structure for intermediate values of the ranges of 

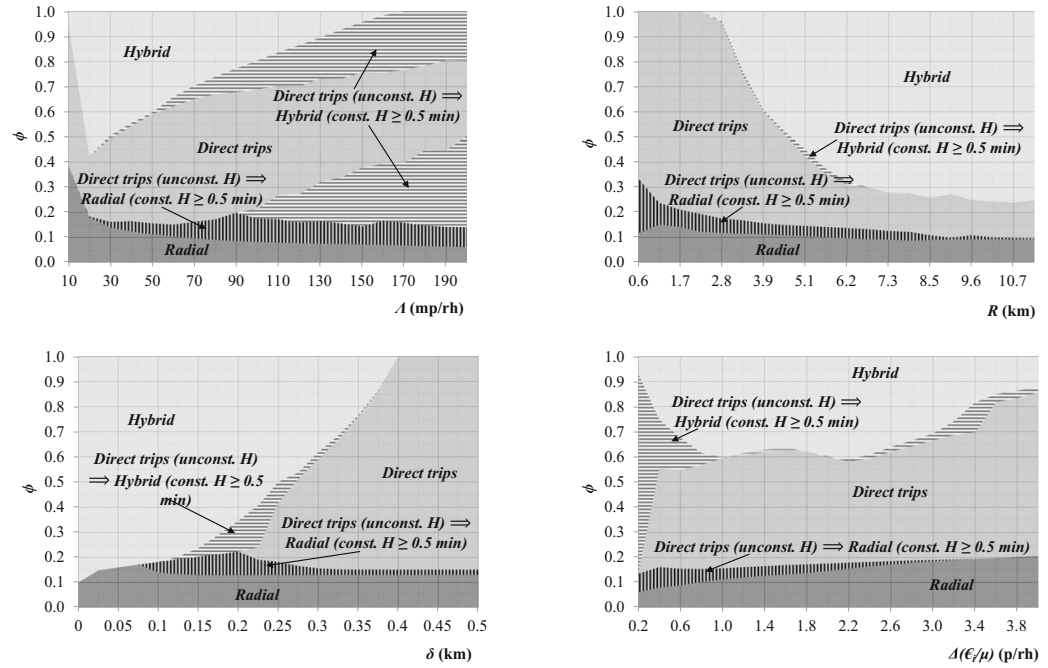

$H y \equiv D T$ (unconst. $H) \Longrightarrow H y$ (const. $H \geq 0.5 \mathrm{~min}$ )

" $D T$ (unconst. $H) \Longrightarrow R a$ (const. $H \geq 0.5 \mathrm{~min}) \quad \| R a$

Fig. 8 Area of applicability with regard to input parameters: demand, city size, transfer penalty, and unit costs

demand and city radius, low transfer penalties, and high agency unit costs. When the comparison is made with a hybrid network, this reduction is greater than $25 \%$ for high levels of demand, small cities, and expensive transfers and agency costs. The directtrip-based structure achieves the maximum savings in those scenarios where its applicability is greater. Compared to the radial network, the saving is between 10 and $20 \%$ in general for $\phi \in[0.15,0.4]$, and compared to the hybrid scheme, the saving is greater than $20 \%$ for small cities, between 15 and $20 \%$ for high demand and expensive transfers, and around 10-15\% with regard to unit costs, especially when $\phi<0.25$ for the four input parameters. Finally, the hybrid network reduces the costs by $5-10 \%$ compared to the other two alternatives for high dispersion in those ranges of input values where this structure gains relevance. There is only one exception: for transfer penalties $\delta<0.1$, the hybrid network achieves savings of 20-25\% compared to direct trips. This last input parameter is the most relevant factor for the competitiveness of a network design based on transfers.

\section{Comparison of Results for Grid and Ring-Radial Street Patterns}

The analysis for a ring-radial street pattern follows the same approach as the case of the grid street pattern in Badia et al. (2016). The assumptions and criteria regarding the choice of user path and derivation of formulations are coincident in both types of cities, allowing us to make a proper comparison between the previous results for the unconstrained headway scenario and the results of Badia et al. (2016). This comparison shows how the street pattern, on which we define the network structures, conditions the guidelines about the transit network design. The main conclusion is that the evolution of the best transit network structure with regard to urban mobility dispersion has the same behavior independently of the street pattern where 

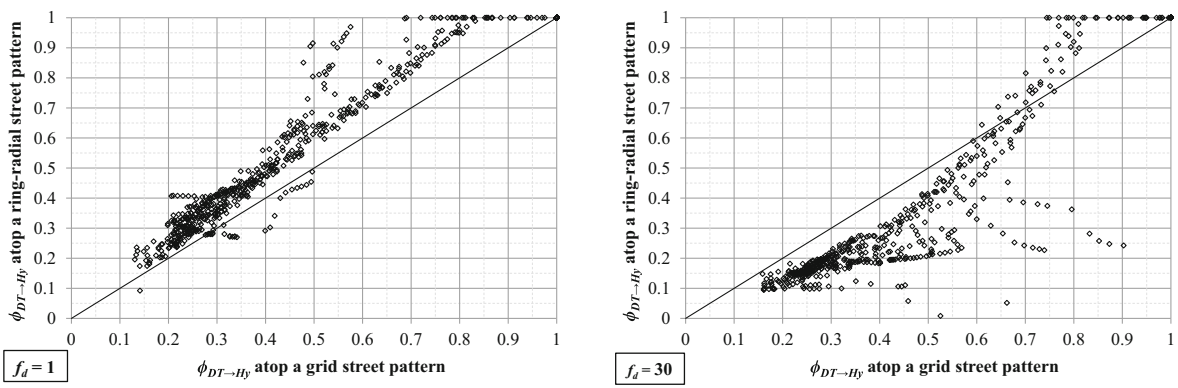

Fig. 9 Comparison between ring-radial and grid street patterns with regard to of the values of $\phi$ that justify the change of structure between direct-trip and transfer-based networks

the network is designed. The area of applicability of each structure varies in a similar way in dependence on changes in the main input parameters of the analytical model. However, the degree of dispersion from which a change between two structures is justified does not have to be coincident in both street patterns. This occurs especially at the boundary between the areas of applicability of direct-tripbased systems and hybrid networks.

Figure 9 compares the value of $\phi$ of the change between direct-trip-based and transfer-based structures for each street layout analyzed. The results are obtained in different scenarios of demand, city size, transfer penalty, and ratio between unit agency costs and value of time. In Fig. 9a, the generated demand is uniformly distributed across the city; that is, $f_{d}=1$. On the other hand, Fig. $9 \mathrm{~b}$ assumes a higher generated demand density in the central area than in the periphery, where $f_{d}$ is 30. Most of the points are above the bisecting line in Fig. 9a and below that line in Fig. 9b. This is a consequence of the worse allocation of resources in ring-radial hybrid networks for more decentralized generated demand. As mentioned in Section 3.2, the peripheral demand in those networks is only supported by radial lines while the circular lines only carry a portion of passengers from the central area. For that reason, the more centralized the generated demand, the better the distribution of users among radial and circular lines. On the other hand, for a grid street pattern, horizontal and vertical lines serve the periphery. Therefore, they always present a good balance of resources invested among them.

Figure 10 makes the same comparison but between a radial scheme and a direct-tripbased network. In this case, we find points above and below the bisecting line in the
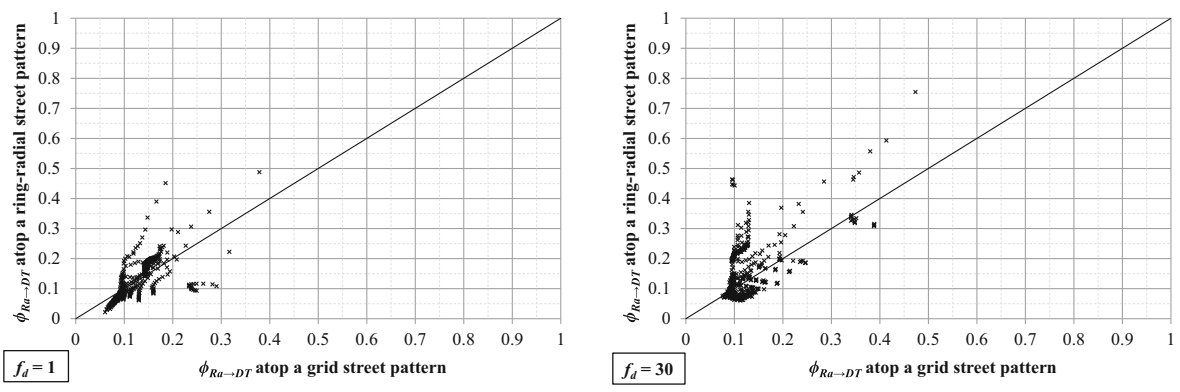

Fig. 10 Comparison between ring-radial and grid street patterns with regard to the values of $\phi$ that justify the change of structure between radial and direct-trip-based networks 
same proportion, and this does not significantly change among different scenarios of generated demand centralization.

\section{Conclusions}

In this study, an analytical model is proposed for the comparison of basic transit network structures when they are designed on a ring-radial street pattern. The main conclusions obtained are in line with the results of Badia et al. (2016): (i) each network structure has its own area of applicability where it is the alternative with the minimum total system cost; (ii) the evolution of the lowest total cost solution with regard to urban dispersion starts with a radial network for low values of dispersion, continues with a direct-trip-based system for intermediate scenarios, and finishes with a hybrid scheme (as a transfer-based structure) where dispersion reaches a higher degree; (iii) between the two parameters related to demand distribution $\phi$ and $f_{d}$, the former is the most determinant in order to discern the optimal structure; and (iv) areas of applicability for each structure are not constant but depend on the characteristics of the city, transport, and demand. Regarding this last point, high levels of demand favor operation using a strategy of direct trips, large cities need synergies among lines by making transfers to achieve a more competitive transit system, and the transfer penalty plays a relevant role in the successful implementation of transfer-based structures. In addition, the radial scheme gains applicability in situations of low demand, small cities, low transfer penalties, and expensive agency costs.

Additionally, in this paper, we consider scenarios where bus stops have a constraint on the minimum service headway. The most sensitive structure to this constraint is the direct-trip based one due to the overlapping lines in the swath-corridors. The previous conclusions do not completely change, but the headway constraint can introduce significant variations in specific scenarios. The main effects on the performance of the direct-trip-based network occur for high levels of demand, losing applicability especially in favor of the hybrid scheme.

The conclusions (i), (ii), and (iv) above are also similar to the outcomes from Fielbaum et al. (2016). A radial network (called a hub-and-spoke network in that paper) is applicable in a city with a strong center, whereas a transfer-based network (feeder-trunk) is more efficient for dispersed cities. Intermediate scenarios of dispersion are the space where direct connections are more competitive. Moreover, their areas of applicability also depend on parameters such as the level of demand and transfer penalty. The higher the values of these parameters, the greater the applicability of direct-trip-based structures in comparison to the other two alternatives. In the same line, Saidi et al. (2017) assess the performance of different metro networks showing that demand patterns or weights of passenger cost parameters condition which is the best network configuration and topology. These coincidences reinforce the conclusions obtained.

On the other hand, the results of Section 4 show that the behavior and general tendencies for the basic structures are independent of the street pattern. The general guidelines about the applicability of each network structure do not change significantly. However, the values of the parameter $\phi$ of change between 
structures are not exactly the same in a specific scenario; their deviations are bounded to 0.08 on average. The greatest variations take place at the boundary between direct trips and transfer-based networks. The main reason is the different behavior of the hybrid network between the two street patterns. In a ring-radial mesh, there is an unbalanced distribution of passengers between radial and circular lines. Only the former serve the periphery; therefore, they carry greater passenger flows. This makes the exploitation of resources less efficient. However, the higher the percentage of central trips, the greater the participation of circular lines. That is, when $f_{d}$ is higher, there is a more balanced demand distribution among lines. As a consequence, the area of applicability of this structure grows.

\section{Future Research}

This paper contributes an analytical formulation to bus network planning. Transport planners can use this tool for the decision-making process. Given a specific city, the model helps analyze what kind of network design approach would be the most competitive option. Therefore, the next step of this research line is how to apply this model in real cities. First of all, we need to measure the real urban dispersion. There are different dimensions of the urban sprawl process. For example, Galster et al. (2001) consider up to eight dimensions, some of them quantified by different indexes. The connection of these measures with the parameters $\phi$ and $f_{d}$ would complete the model, leading to its applicability in real scenarios.

Application in real cities will allow us to check the similarities between the metrics of the model with its simple spatial distribution of demand and the metrics in the city with a real origin-destination matrix. Previous studies have used this family of models for the design of real bus networks. Estrada et al. (2011) is an example where the Barcelona bus network is redesigned. That paper shows that the main discrepancy between user metrics is the trip length, which is longer in the model due to an overestimation of peripheral trips. The real demand distribution is more centralized than the idealized uniform distribution over the whole city assumed in the initial model (Daganzo 2010). However, the introduction of parameters $\phi$ and $f_{d}$ reduces that overestimation since the model can consider scenarios with a more concentrated demand around the center. This upgrade of the initial model would improve the correspondence between theoretical and real metrics, in particular, obtaining a better approximation of trip length.

On the other hand, additional aspects could be considered in the model. One of these aspects is the introduction of a demand function that depends on the characteristics of the network design. In this way, we can analyze possible effects on demand behavior according to how each network structure works. In the same line, a more complex demand distribution with destinations outside the central attractant area could provide additional insights about the competitiveness and applicability of each network structure. Another interesting factor is the congestion in the transit system due to the arrangement of lines. The critical congested points on bus lines are at stops (TRB 2003), where low headways can generate long queues of buses waiting for loading passengers. The constraint on the service headway shows that congestion has an 
important effect in some scenarios. In this paper, we consider the same minimum headway for all the structures. However, different operating measures could be implemented in each structure. In a hybrid scheme, each street is served by only one line, then all its vehicles should serve the same stops. On the other hand, the swath-corridors of the direct-trip-based structure are crossed by different lines that work independently to each other. In this way, they could be allocated to different stops, increasing the capacity of these corridors.

Finally, this model considers simple transit systems that only work with one network structure. However, if more complex demand representations are assumed, the analysis of hierarchical systems would be interesting (Fan et al. 2018; Van Nes 2002). Mixed structures could better satisfy the diverse mobility requirements. For example, the most demanded zones can be connected with the whole city by direct services, while the rest of the trips would be completed by a base network where transfers would be an essential step of their paths.

Acknowledgments The author was partially funded by an FPU grant, reference number AP2010-4544, from the Spanish Ministry of Education.

Funding Information Open access funding provided by Royal Institute of Technology.

\section{Compliance with ethical standards}

Conflict of interest On behalf of all authors, the corresponding author states that there is no conflict of interest.

\section{Appendix 1. Formulation of the model}

Nomenclature $H$, Headway [h]; $\theta$, Ring stop spacing or radial line spacing [rad]; $s$, Radial stop spacing or ring line spacing $[\mathrm{km}] ; d$, Swath width or swath-corridor spacing in a direct-trip-based network structure $[\mathrm{km}] ; \alpha$, Variable defining the central ring-radial mesh in a hybrid network structure [-]; $Z$, Total system cost $[\mathrm{h} / \mathrm{p}-\mathrm{h}] ; C_{A}$, Agency cost $[\mathrm{h} / \mathrm{p}-\mathrm{h}] ; C_{U}$, User cost $[\mathrm{h} / \mathrm{p}-\mathrm{h}] ; L$, Infrastructure length $[\mathrm{km}] ;$, Kilometers travelled per vehicle and hour $[\mathrm{veh}-\mathrm{km} / \mathrm{h}] ; M$, Number of vehicles working per hour [veh-h/h]; A, Average access time per trip [h]; $W$, Average waiting time per trip $[\mathrm{h}]\left(W_{c}\right.$ in the central area and $W_{p}$ in the periphery); $T$, Average in-vehicle time per trip [h]; $E$, Average in-vehicle distance per trip $[\mathrm{km}]\left(E_{c}\right.$ in the central area and $E_{p}$ in the periphery); $v_{c}$, Commercial speed $[\mathrm{km} / \mathrm{h}] ; l_{s}$, Average infrastructure length per stop $[\mathrm{km}] ; e_{T}$, Average number of transfers per trip $[-] ; O$, Vehicle occupancy at the most loaded points of the network [p/veh]; $\phi$, Demand decentralization degree $[-] ; f_{d}$, Factor of densities between central and peripheral areas $[-] ; \rho$, Portion of generated demand in the central area $[-] ; \Lambda$, Demand during the rush hour $[\mathrm{p} / \mathrm{h}] ; \lambda$, Average hourly demand $[\mathrm{p} / \mathrm{h}] ; R$, Radius of the circle that represents the city $[\mathrm{km}] ; \mu$, Value of time $[€ / \mathrm{h}] ; \delta$, Equivalent penalty distance per transfer $[\mathrm{km}] ; v$, Cruising speed (without considering time spent at stops) $[\mathrm{km} / \mathrm{h}] ; w$, Pedestrian speed $[\mathrm{km} / \mathrm{h}] ; \epsilon_{i}$, Unit agency cost, where $i=L, V$, or $M[€ / \mathrm{km}-\mathrm{h} ; € / \mathrm{veh}-\mathrm{km} ; € / \mathrm{veh}-\mathrm{h}] ; C$, Vehicle capacity [p/veh]; $S F$, Occupancy safety factor $[-] ; \tau$, Dwell time per stop [h]; $\tau^{\prime}$, Boarding (and alighting) time per passenger $[\mathrm{h}] ; w_{j}$, Time perception weight, where $j=A, W, T$, or $t[-] ; H_{\text {min }}$, Minimum service headway constraint [h]; $H_{s}$, Cut-off headway between types of service $[\mathrm{h}] ; h_{s}$, Safety waiting time $[\mathrm{h}] ; f_{s}$, Home waiting time factor [-]; $\varepsilon_{H}$, Parameter that defines the boundary between both types of service operations in a direct-trip-based network structure [-]; $O_{i}$, Origin of trip; $D_{i}$, Destination of trip; $C P_{i}$, Critical point of occupancy 
This appendix includes explanations and proofs in order to demonstrate the estimation of the different partial costs for the different network structures. The next process of derivation is based on the hybrid network model presented in Badia et al. (2014). For this reason, only some indications are included in this appendix with regard to the formulation of the hybrid scheme and the radial network. More detailed explanations are shown for the direct-trip-based structure.

\section{Hybrid Network Structure}

The network configuration and the level of service are the same as in Badia et al. (2014) for $s=s_{r}$ and $\theta=\theta_{c}$. That is, intermediate stops between transfer-stops in the central mesh are not considered in this paper; all of the central stops are served by one radial line and one circular line. Then, the estimations of agency costs in Table 1 are exactly the same as in Badia et al. (2014). For that reason, their derivations are omitted.

The estimations of user costs differ from the previous paper due to the different spatial distributions of demand. This changes the weighting between the central area and the periphery. In this case, there are six types of trips in function of the origin and destination locations. Three of them have the destination in the central area that is served by the ring-radial mesh, and they are distinguished by the location of the origin: (a.1) the origin in the same area, whose probability is $\rho \alpha^{4} / \phi^{4}$, (a.2) the origin in the remaining central area, whose probability is $\rho \alpha^{2}\left(\phi^{2}-\alpha^{2}\right) / \phi^{4}$, or (a.3) the origin in the periphery, whose probability is $(1-\rho) \alpha^{2} / \phi^{2}$. On the other hand, the other three types of trips have the destination in the central area that is only served by radial lines and the origin is located in: (a.4) the central ring-radial mesh, whose probability is $\rho \alpha^{2}\left(\phi^{2}-\alpha^{2}\right) / \phi^{4},($ a.5) the central area only served by radial lines, whose probability is $\rho\left(\phi^{2}-\alpha^{2}\right)^{2} / \phi^{4}$, or (a.6) the periphery, whose probability is $(1-\rho)\left(\phi^{2}-\alpha^{2}\right) / \phi^{2}$. Assuming the weight of each trip category, the process of derivation is very similar. Then, the reader is invited to check these derivations in Badia et al. (2014). Since the estimations have been improved, only two metrics are derived in detail here: access cost and maximum occupancy.

- Result 1. The expected walking time at the origin and destination is $A=\left[\alpha R \theta\left(6 \phi^{2}\right.\right.$ $\left.\left.-\alpha^{2}-\rho \alpha^{2}\right) / 6 \phi^{2}+s\right] / 2 w$

- Proof. It is assumed that every user connects to the system at his or her nearest stop. The average distance to one stop is the mean of the walking distances of the closest user and the furthest one. The former user is located at the stop itself, which implies a null distance. The walking distance in the latter case is decomposed into two sections: one radial path whose length is $s / 2$, which is constant across the central mesh; the other a transverse path that varies with the distance from the city center with a ratio of $\theta / 2$. Therefore, the average walking distance to a stop at a cordon $\beta R$ is $\beta R \theta / 4+s / 4$. As $\beta$ follows a triangular probability density function $2 \beta / \alpha^{2}$ in the central mesh, the resultant average walking distance in that area is $\int_{0}^{\alpha}\left(2 \beta / \alpha^{2}\right)(\beta R \theta / 4+s / 4) d \beta=\alpha R \theta / 6+s / 4$. In the branched section of the radial lines outside the central mesh, the accessibility is constant and equal to the boundary $\alpha R$. Therefore, this average access distance is $\alpha R \theta / 4+s / 4$.

On the other hand, the egress cost is the same as the access cost. The total walking distance is the result of weighting each of the aforementioned distances by 
the portion of trips generated and attracted in the central mesh and in the periphery. That distance is $(\alpha R \theta / 6+s / 4)(1+\rho) \alpha^{2} / \phi^{2}+(\alpha R \theta / 4+s / 4)(1+\rho)\left(\phi^{2}-\alpha^{2}\right) / \phi^{2}+$ $(\alpha R \theta / 4+s / 4)(1-\rho)=\alpha R \theta\left(6 \phi^{2}-\alpha^{2}-\rho \alpha^{2}\right) / 12 \phi^{2}+s / 2$. Finally, the expected walking time is the previous total distance divided by the pedestrian speed $w$.

- Result 2. The expected vehicle occupancy at the critical load point during the rush hour is $O=(S F) \max \left\{O_{r p} ; O_{c p} ; O_{r c}\right.$, if $r_{r}<\alpha R ; O_{c c}$, if $\left.r_{c}<\alpha R\right\}$

- Proof. The vehicle occupancy is studied at those critical points of the system that present the highest passenger loads. Figure $4 \mathrm{c}$ shows the locations of those points in this network. Two possible locations exist: at an inner point of the ring-radial mesh and on the boundary of that mesh. In addition, as two types of lines compose the network, we distinguish the occupancy of radial lines and circular lines. As the curves of generated and attracted demand are different, the occupancy in one direction can be different from that in the other direction. It is easy to check that the generated demand produces higher loads in radial lines than the attracted demand. The percentage of generated demand outside the mesh is greater than the percentage of attracted demand, and that demand is only carried by radial lines. However, with regard to circular lines, the attraction determines the highest occupancy for the same reason. Circular lines only serve inside the central mesh where there are more attracted trips than generated ones.

Boundary of ring-radial mesh: The demand that crosses this limit through the radial corridors in the rush hour coincides with the demand generated outside the central mesh: in the intermediate area it is $\Lambda \rho\left(\phi^{2}-\alpha^{2}\right) / \phi^{2}$ and in the external periphery $\Lambda(1-\rho)$. This demand is allocated to the existing corridors, whose number is $2 \pi / \theta$. As corridors are branched irregularly in the periphery, Badia et al. (2014) show that some of them serve a peripheral area approximately twice as large as the areas served by other corridors. For this reason, that demand is multiplied by a factor of two. Finally, this demand is assigned to the number of vehicles providing the service $(1 / H)$, obtaining the following occupancy: $O_{r p}=H \theta \Lambda\left(1-\rho \alpha^{2} / \phi^{2}\right) / \pi$. Regarding the circular border corridor, the maximum occupancy that a vehicle supports is obtained from the number of trips generated in the area of influence of this corridor and the rest of the periphery in an arc of $2 \mathrm{rad}$, whose destination is situated at an average of $1 \mathrm{rad}$ from the analyzed point. Obviously, this demand must be redistributed by the number of vehicles $(1 / H)$, giving an occupancy $O_{c p}=H \Lambda\left[4 R^{2}\left(\phi^{2}-\rho \alpha^{2}\right)+\rho s(4 \alpha R-s)\right]\left[4 R^{2}\left(\phi^{2}-\alpha^{2}\right)+s(4 \alpha R-s)\right] /$ $32 \pi^{2} \phi^{4} R^{4}$.

Inside the ring-radial mesh: The number of passengers crossing a point of a radial line at a distance from the city center $r_{r}$ is all the demand generated in the area of influence of the radial line beyond that point and attracted inside the cordon where the point is located and outside that cordon if the destination is at an angular spacing of more than $2 \mathrm{rad}$ from that radial line. The former demand is $\theta \Lambda\left[\left(\rho \alpha^{2} / \phi^{2}\right)\left(\pi \alpha^{2} R^{2}-\pi r_{r}^{2}\right) / \pi \alpha^{2} R^{2}+2\left(\rho\left(\phi^{2}-\alpha^{2}\right) / \phi^{2}+(1-\rho)\right)\right] / 2 \pi$, and the latter is $\theta \Lambda\left[(1-2 / \pi)\left(\pi \phi^{2} R^{2}-\pi r_{r}^{2}\right) / \pi \phi^{2} R^{2}+\pi r_{r}^{2} / \pi \phi^{2} R^{2}\right] / 2 \pi$. The value of $r_{r}$ that gives the maximum occupancy is $R \sqrt{\rho\left(4 \phi^{2}+2 \rho \phi^{2}-\pi \rho \phi^{2}-2 \rho \alpha^{2}\right)} / 2 \rho$. Finally, allocating the passengers to the vehicles that serve the line $(1 / H)$, we obtain the maximum occupancy at the inner point of a radial line $O_{r c}=H \theta \Lambda\left[4 \phi^{2}+\rho\left(\pi \phi^{2}-2 \phi^{2}\right.\right.$ 
$\left.\left.-2 \alpha^{2}\right)\right]^{2} / 16 \pi^{2} \phi^{4} \rho$. On the other hand, a circular line at a distance $r_{c}$ from the city center carries all the demand attracted in its surroundings in an arc length of $2 \mathrm{rad}$ and is generated outside the cordon where the line is located. That is, $\left(2 \Lambda s r_{c} / \pi \phi^{2} R^{2}\right)\left[4 \phi^{2} R^{2}\right.$ $\left.-\rho\left(2 r_{c}-s\right)^{2}\right] / 8 \pi \phi^{2} R^{2}$. Then, the most loaded line is located at a radius $r_{c}=s / 3+\sqrt{12 \rho \phi^{2} R^{2}+\rho^{2} s^{2}} / 6 \rho$. As the line is served by $1 / H$ vehicles per hour, the most occupied vehicle in a circular line carries $O_{c c}=H \Lambda s\left(2 \rho s+\sqrt{\rho\left(12 \phi^{2} R^{2}+\rho s^{2}\right)}\right)\left(12 \phi^{2} R^{2}+s \sqrt{\rho\left(12 \phi^{2} R^{2}+\rho s^{2}\right)}-\rho s^{2}\right) / 108 \pi^{2} \phi^{4} R^{4} \rho$.

Finally, from the different analyzed points of the system, the point that presents the greatest occupancy is the determinant in the model's results. However, whether $r_{r}$ or $r_{c}$ is located outside the central mesh, the inner points would be at the boundary. Therefore, we only consider $O_{r p}$ and $O_{c p}$. Furthermore, the rush hour also has its own peaks, and for this reason, the occupancy is increased by a safety factor $(S F)$.

\section{Radial Network Structure}

The radial structure is the hybrid scheme for $\alpha=0$. It is only composed of radial lines gathered in the central point of the city and branched as they run away from the center. The main difference from the hybrid network is that there are no circular lines in this case. Therefore, its costs in Tables 1 and 2 are estimated in the same way as in the peripheral band of the previous structure. To derive them, we have to replace $\alpha R$ by $s$ since the reference point here is the first point of ramification. However, the number of transfers is estimated with more accuracy. This aspect also conditions the waiting and in-vehicle times since the probability of zero and onetransfer trips varies, although the derivations follow the same steps. Therefore, the number of transfers is explained in detail but the remaining costs can be understood from the hybrid scheme in Badia et al. (2014). In addition, the occupancy is also explained in this appendix. First of all, there are only two types of trips in this network: (b.1) trips with the origin in the central attractant area, whose probability is $\rho$, and (b.2) trips with the origin in the periphery outside that central area, whose probability is the complement, $(1-\rho)$.

- Result 3. The expected number of transfers per trip is $e_{T}=1-2 s \theta(3+\rho) / 3 \pi \phi R$

- Proof. A direct trip exists when the origin and destination are located in the area of influence of the same line. Due to the line branching, the more central the stop is, the greater the number of lines that serve that stop. Therefore, the probability of direct trips between two cordons is determined by the most central one. Given two cordons $\beta_{O} D$ and $\beta_{D} D$, if the former is greater, that probability is $\theta s / \pi \beta_{O} D$; otherwise, $\theta s / \pi \beta_{D} D$. This probability is the angle between two stops at that cordon $\theta s / \beta D$ divided by the total angle $2 \pi$ and multiplied by two since the line crosses the city from one extreme to another through the city center. Knowing that the p.d.f. of $\beta_{O}$ and $\beta_{D}$ is $2 \beta_{i} / \phi^{2}$ in the central area, the probability of zero-transfer trips of category (b.1) is $\int_{0}^{\phi}\left(2 \beta_{O} / \phi^{2}\right)\left[\int_{0}^{\beta_{O}}\left(2 \beta_{D} / \phi^{2}\right)\left(\theta s / \pi \beta_{D} R\right) d \beta_{D}+\int_{\beta_{O}}^{\phi}\left(2 \beta_{D} / \phi^{2}\right)\right.$ $\left.\left(\theta s / \pi \beta_{O} R\right) d \beta_{D}\right] d \beta_{O}=8 s \theta / 3 \pi \phi R$. If the origin is peripheral, that probability is 
always determined by the destination. Therefore, $\int_{0}^{\phi}\left(2 \beta_{D} / \phi^{2}\right)\left(\theta s / \pi \beta_{D} R\right) d \beta_{D}=2$ $s \theta / \pi \phi R$ is the probability of direct trips in category (b.2). Finally, weighting by each trip category, we obtain the portion of direct trips in this network structure $P_{0}=2 s \theta(3+\rho) / 3 \pi \phi R$. For the remainder of the trips, one transfer is required. The total probability theorem gives the probability of one-transfer trips, which is coincident with the expected number of transfers per trip $e_{T}$.

- Result 4. The expected vehicle occupancy at the critical load point during the rush hour is $O=(S F) H \Lambda \theta[3 \pi \phi R-\theta s(3+\rho)] / 6 \pi^{2} \phi R$

- Proof. Figure 4a shows that the most loaded point of this structure is the central point of the city. All passengers who make a transfer go through that center. In addition, half of the direct trips do too, as mentioned above in Result 3. Therefore, the number of passengers during the rush hour at that point is $\Lambda\left[1-(3+\rho) \theta_{s} /\right.$ $3 \pi \phi R]$. All of this demand is allocated along the different corridors of the city center $2 \pi / \theta$ and the vehicles that serve each of them $(1 / H)$. Therefore, the maximum vehicle occupancy is $H \Lambda \theta[3 \pi \phi R-(3+\rho) \theta s] / 6 \pi^{2} \phi R$. As in the other structures, this value is penalized by the factor $(S F)$.

\section{Direct-Trip-Based Network Structure}

From the explanations in Sections 2.2 and 2.4, we now start the derivation of the formulas used to calculate the costs of this network structure. This derivation is similar to that of Badia et al. (2016), where this structure is designed for a grid street pattern. Figures $2 \mathrm{~b}$ and 3 help to understand in detail the arrangement of the lines on the ring-radial street pattern. The different swathcorridors are located at a distance $i \cdot d$ from the city center, with $i=1,2, \ldots, n$, where $n$ is the number of swaths $(2 \phi R-d) / 2 d$ without considering the central one. A relevant change from the previous structures is the possibility of two types of services: according to headways or schedules. This divides the city into two areas at the cordon $\varepsilon_{H} R$. The system operates according to headways inside this cordon and according to schedules outside it. The value of $\varepsilon_{H}$ is $H_{s} s / H R$, the cordon where the service headway reaches the cut-off headway $H_{s}$. We accept operating according to schedules in this structure since transfers do not exist; that is, $e_{T}$ is zero. Finally, in this structure, we distinguish the same two categories of trips as in the radial network: (b.1) and (b.2).

- Result 5. The total length of the two-way infrastructure system is $L=\pi[(4+$ $\left.\theta) R(R+s)-2 \theta s^{2}\right] / 4 s \theta+\pi\left(4 \phi^{2} R^{2}-d^{2}\right) / 4 d$

- Proof. This length is the result of adding the swath-corridors' lengths to the initial radial network in Table 1. One swath-corridor is a circumference of radius $i \cdot d$. Therefore, the additional length is $\sum_{1}^{(2 \phi R-d) / 2 d} 2 \pi i d=\pi\left(4 \phi^{2} R^{2}-d^{2}\right) / 4 d$.

- Result 6. The total vehicle-distance travelled per hour is $V=\pi\left[4 \phi R^{2}((4+\theta)(2-\right.$ $\phi)+2 \pi \phi)-d(2 d(\pi-2)-\theta(d-4 s))] / 4 d \theta H$ 
- Proof. We add the vehicle-kilometers of the remainder of the swaths to those covered by the radial lines that serve the central swath. One line that serves a swath at a cordon $i \cdot d$ runs from the city edge to its swath-corridor, covering a length of $((4+\theta) s / 4)((R-i d) / s)$, along that corridor for a length of $\pi i d$, and finally, from that swath-corridor to the city edge, covering ( $4+$ $\theta) s / 4)((R-i d) / s)$ units of distance. The sum of these lengths is multiplied by two due to its bidirectionality. As the number of vehicles that serves a swath is $2 \pi / \theta H$, the number of kilometers travelled in that swath is $2 \pi[(4+$ $\theta) R+(2 \pi-4-\theta) i d] / \theta H$. Adding all the swaths, we obtain $\pi(2 \phi R-d)[2 R(8+$ $2(\pi-2) \phi+(2-\phi) \theta)+d(2 \pi-4-\theta)] / 4 d \theta H$. The resultant number of kilometers travelled is the total sum of the radial network and the remainder of the swaths, $V=\pi\left[4 \phi R^{2}((4+\theta)(2-\phi)+2 \pi \phi)-d(2 d(\pi-2)-\theta(d-4 s))\right] / 4 d \theta H$.

- Result 7. The expected commercial speed during the rush hour is $v_{c}=1 /\left[1 / v+\tau / l_{s}+\right.$ $\left.\tau^{\prime} \Lambda / V\right]=1 /\left[1 / \nu+\tau \pi\left[4 d R(R+s)+s\left(4 \phi^{2} R^{2}-d^{2}\right)\right] / 4 L \theta d s^{2}+\tau^{\prime} \Lambda / V\right]$

- Proof. In this case, the number of stops is $2 \pi R(R+s) / \theta s^{2}+\pi\left(4 \phi^{2} R^{2}-d^{2}\right) / 2 \theta d s$ and the average length per stop $l_{S}$ is the total infrastructure length $2 L$ divided by that number of stops. On the other hand, no transfers exist; that is, $e_{T}=0$.

- Result 8. The expected walking time at the origin and destination is $A=[s(1+$ $2 \theta)+d] / 4 w$

- Proof. The access distance at the beginning of the trip is equal to the access distance in the radial network structure $s(1+\theta) / 4$. However, the egress distance is not coincident with the egress distance in that radial network structure. In this case, the final stop is in one swath-corridor. The width of one swath is $d$, and therefore, the user walks a quarter of that width in the radial direction on average. In the ring direction, the walking distance is $\theta s / 4$ since the stop spacing is $\theta s$. Finally, adding these distances and dividing by the pedestrian speed, the total access cost is $[s(1+$ $2 \theta)+d] / 4 w$.

- Result 9. The expected waiting time per user at the origin stop is:

$$
\begin{aligned}
& \text { if } \varepsilon H \geq 1 \quad W=H[5(1-\rho)+\phi(1+\phi)(5+\rho)] R / 15(1+\phi) s \\
& \text { if } \phi \leq \varepsilon H<1 \quad W=\left[h_{s}\left(1-\varepsilon_{H}^{2}\right)+H\left(2 f_{s}\left(1-\varepsilon_{H}^{3}\right)+\left(\varepsilon_{H}^{3}-\phi^{3}\right)\right) R / 3 s\right] \\
& (1-\rho) /\left(1-\phi^{2}\right)+2 \phi R H \rho / 5 s \\
& \text { if } \varepsilon H<\phi \quad W=\left[h_{s}+2 f_{s}\left(1+\phi+\phi^{2}\right) R H / 3(1+\phi) s\right](1-\rho) \\
& +\left[h_{s}\left(\phi^{4}-\varepsilon_{H}^{4}\right)+2 H\left(2 f_{s}\left(\phi^{5}-\varepsilon_{H}^{5}\right)+\varepsilon_{H}^{5}\right) R / 5 s\right] \rho / \phi^{4}
\end{aligned}
$$

- Proof. Two aspects are highlighted. First, the service headway at one stop increases with the distance from the city center due to the line branching. At a cordon $\beta R$, the headway is $\beta R H / s$ as in the radial network structure or the external area in the hybrid network. Secondly, as all of the trips are direct, the system can operate according to headways and schedules. The former implies a waiting time equivalent 
to half of the headway, and the waiting time in the latter follows Eq. (3) in Section 2.4.2.

Following a similar approximation to Badia et al. (2016), parameter $\varepsilon_{H}$ determines the cordon $\varepsilon_{H} R$ that delimits a central area where the system operates according to headways and the external one where it operates according to schedules. Headways inside that boundary are lower than $H_{s}$; therefore, $\varepsilon_{H}=H_{s} s / H D$. According to the value of that parameter, we identify three different scenarios: (S.1) when $\varepsilon_{H} \geq 1$, the system only operates according to headways; (S.2) when $\phi \leq \varepsilon_{H}<$ 1 , the most external periphery operates according to schedules; and (S.3) when $\varepsilon_{H}$ $<\phi$, the system only operates according to headways in the most internal region of the central attractant area. In the first scenario, the city is divided into the same two areas as in the rest of the proofs, with two types of trips, (b.1) and (b.2). However, in the other two scenarios, another area appears. In (S.2), the peripheral area related to (b.2) is divided into two subareas, one external, whose probability is $\left(1-\varepsilon_{H}^{2}\right)(1-\rho) /\left(1-\phi^{2}\right)$, and other internal one, whose probability is $\left(\varepsilon_{H}^{2}-\phi^{2}\right)(1-\rho) /\left(1-\phi^{2}\right)$. In this scenario, the central area is (b.1). In (S.3), the periphery is (b.2), but the central area is divided into two, of which the external subarea has a probability of $\left(\phi^{2}-\varepsilon_{H}^{2}\right) \rho / \phi^{2}$ and the internal subarea has a probability of $\varepsilon_{H}^{2} \rho / \phi^{2}$.

Focusing our attention on (S.1), the average headways of the central attractant area and the periphery are weighted by the headway of each cordon $\beta D$. The p.d.f. of $\beta$ is triangular and follows the functions $2 \beta / \phi^{2}$ and $2 \beta /\left(1-\phi^{2}\right)$ in the central attractant area and in the periphery respectively. Therefore, those average headways are $H_{c}=\int_{0}^{\phi}\left(2 \beta_{O} / \phi^{2}\right)\left[\int_{0}^{\beta_{O}}\left[\left(2 \beta_{D} / \phi^{2}\right)\left(\beta_{O} R / s\right) H\right] d \beta_{D}+\int_{\beta_{O}}^{\phi}\left[\left(2 \beta_{D} / \phi^{2}\right)\left(\beta_{D} R / s\right) H\right]\right.$ $\left.d \beta_{D}\right] d \beta_{O}=4 \phi R H / 5 s$ and $H_{p}=\int_{\phi}^{1}\left[\left(2 \beta /\left(1-\phi^{2}\right)\right)(\beta R / s) H\right] d \beta=2\left(1+\phi+\phi^{2}\right)$ $R H / 3(1+\phi) s$. This gives an expected waiting time $W=\left[H_{c} \rho+H_{p}(1-\rho)\right] / 2=$ $H[5(1-\rho)+\phi(1+\phi)(5+\rho)] R / 15(1+\phi) s$.

The same derivation is done for the other two scenarios. In (S.2), the average headways in the two subareas of the periphery are $H_{p}^{o}=\int_{\varepsilon_{H}}^{1}\left[\left(2 \beta /\left(1-\varepsilon_{H}^{2}\right)\right)(\beta R / s)\right.$ $H] d \beta=2\left(1+\varepsilon_{H}+\varepsilon_{H}^{2}\right) R H / 3\left(1+\varepsilon_{H}\right) s$ and $H_{p}^{i}=\int_{\phi}^{\varepsilon_{H}}\left[\left(2 \beta /\left(\varepsilon_{H}^{2}-\phi^{2}\right)\right)(\beta R / s) H\right]$ $d \beta=2\left(\varepsilon_{H}^{2}+\varepsilon_{H} \phi+\phi^{2}\right) R H / 3\left(\varepsilon_{H}+\phi\right) s$. In (S.3), the average headways for the three different categories of trips between the two central subareas are $H_{c}^{o}=\int_{\varepsilon_{H}}^{\phi}$ $\left(2 \beta_{O} /\left(\phi^{2}-\varepsilon_{H}^{2}\right)\right)\left[\int_{\varepsilon_{H}}^{\beta_{O}}\left[\left(2 \beta_{D} /\left(\phi^{2}-\varepsilon_{H}^{2}\right)\right)\left(\beta_{O} D / s\right) H\right] d \beta_{D}+\int_{\beta_{O}}^{\phi}\left[\left(2 \beta_{D} /\left(\phi^{2}-\varepsilon_{H}^{2}\right)\right)\right.\right.$ $\left.\left.\left(\beta_{D} D / s\right) H\right] d \beta_{D}\right] d \beta_{O}=4\left(2 \varepsilon_{H}^{3}+4 \varepsilon_{H}^{2} \phi+6 \varepsilon_{H} \phi^{2}+3 \phi^{3}\right) R H / 15\left(\varepsilon_{H}+\phi\right)^{2} s$, $H_{c}^{i, o}=\int_{\varepsilon_{H}}^{\phi}\left[\left(2 \beta /\left(\phi^{2}-\varepsilon_{H}^{2}\right)\right)(\beta D / s) H\right] d \beta=2\left(\phi^{2}+\phi \varepsilon_{H}+\varepsilon_{H}^{2}\right) D H / 3\left(\varepsilon_{H}+\phi\right) s$, a n d f i n a $11 \mathrm{y} \quad H_{c}^{i}=\int_{0}^{\varepsilon_{H}}\left(2 \beta_{O} / \varepsilon_{H}^{2}\right)\left[\int_{0}^{\beta_{O}}\left[\left(2 \beta_{D} / \varepsilon_{H}^{2}\right)\left(\beta_{O} R / s\right) H\right] d \beta_{D}+\int_{\beta_{O}}^{\varepsilon_{H}}\right.$ $\left.\left[\left(2 \beta_{D} / \varepsilon_{H}^{2}\right)\left(\beta_{D} R / s\right) H\right] d \beta_{D}\right] d \beta_{O}=4 \varepsilon_{H} D H / 5 s$. Then, the resultant waiting times for these scenarios are as follows: 


$$
\begin{aligned}
& \text { if } \phi \leq \varepsilon H<1 \quad W=\left[\left(h_{s}+f_{s} H_{p}^{o}\right)\left(1-\varepsilon_{H}^{2}\right)+H_{p}^{i}\left(\varepsilon_{H}^{2}-\phi^{2}\right) / 2\right](1-\rho) /\left(1-\phi^{2}\right)+\rho H_{c} \phi^{2} / 2= \\
& {\left[h_{s}\left(1-\varepsilon_{H}^{2}\right)+H\left(2 f_{s}\left(1-\varepsilon_{H}^{3}\right)+\left(\varepsilon_{H}^{3}-\phi^{3}\right)\right) R / 3 s\right](1-\rho) /\left(1-\phi^{2}\right)+2 \phi R H \rho / 5 s} \\
& \text { if } \varepsilon H<\phi \quad W=\left(h_{s}+f_{s} H_{p}\right)(1-\rho)+\left[\left(h_{s}+f_{s} H_{c}^{o}\right)\left(\phi^{2}-\varepsilon_{H}^{2}\right)^{2}+\left(h_{s}+f_{s} H_{c}^{i, o}\right) 2 \varepsilon_{H}^{2}\left(\phi^{2}-\varepsilon_{H}^{2}\right)+H_{c}^{i} \varepsilon_{H}^{4} / 2\right] \\
& \rho / \phi^{4}=\left[h_{s}+2 f_{s}\left(1+\phi+\phi^{2}\right) R H / 3(1+\phi) s\right](1-\rho)+ \\
& {\left[h_{s}\left(\phi^{4}-\varepsilon_{H}^{4}\right)+2 H\left(2 f_{s}\left(\phi^{5}-\varepsilon_{H}^{5}\right)+\varepsilon_{H}^{5}\right) R / 5 s\right] \rho / \phi^{4}}
\end{aligned}
$$

- Result 10. The expected in-vehicle travel time per trip is $T=E / v_{c}=[5(4+\theta)(1$ $-\rho)+2 \phi(1+\phi)(5 \pi+\rho(4+\theta-\pi))] R / 30(1+\phi) v_{c}$.

- Proof. Like the line length, a trip can also be divided into two parts: one in the swath-corridor and the other in the branched section of the lines. This second section follows the same path as the radial network. This goes from the most external extreme of the trip to the cordon where the other extreme is located. Its length is $\left|\beta_{O}-\beta_{D}\right| R$ units of distance in the radial direction and $(\theta s / 4)\left|\beta_{O}-\beta_{D}\right| R / s$ in the circular direction. The section in the swath-corridor runs from the entrance point of the previous section to the other extreme of the trip. The distance travelled in this case is, on average, a quarter of the cordon length, $2 \pi\left(\min \left\{\beta_{O} ; \beta_{D}\right\}\right) R / 4$. With this information, we can estimate the distance travelled on the peripheral trips as $E_{p}$ $=\int_{\phi}^{1}\left(2 \beta_{O} /\left(1-\phi^{2}\right)\right)\left(\beta_{O}-\phi\right) R(1+\theta / 4) d \beta_{O}+\int_{0}^{\phi}\left(2 \beta_{D} / \phi^{2}\right)\left[\left(\phi-\beta_{D}\right) R(1+\theta / 4)+\right.$ $\left.\pi \beta_{D} R / 2\right] d \beta_{D}=\left(4+\theta+2 \pi \phi+2 \pi \phi^{2}\right) R / 6(1+\phi)$ and that on the central trips as $E_{c}=\int_{0}^{\phi} \int_{0}^{\phi}\left(2 \beta_{D} / \phi^{2}\right)\left(2 \beta_{O} / \phi^{2}\right)\left[\left|\beta_{O}-\beta_{D}\right| R(1+\theta / 4)+\pi \min \left\{\beta_{O} ; \beta_{D}\right\} R / 2\right] d \beta_{O} d \beta_{D}$ $=(4 \pi+4+\theta) \phi R / 15$. Finally, weighting these two lengths by the probability of each trip category, the expected in-vehicle travel distance per trip $E$ is obtained. Knowing $E$ and dividing it by the commercial speed $v_{c}$, the in-vehicle travel time $T$ is determined.

- Result 11. The expected vehicle occupancy at the critical load point during the rush hour is:

$$
\begin{aligned}
& \text { if }\left(2 d \rho+\sqrt{12 \phi^{2} R^{2} \rho+d^{2} \rho^{2}}\right) / 6 \rho \leq \phi R, \quad O=(S F) H \Lambda \theta d\left[\begin{array}{c}
d \rho\left(36 \phi^{2} R^{2}-\rho d^{2}\right)+ \\
\rho^{1 / 2}\left(12 \phi^{2} R^{2}+\rho d^{2}\right)^{3 / 2}
\end{array}\right] / 216 \pi \phi^{4} R^{4} \rho \\
& \text { if }\left(2 d \rho+\sqrt{12 \phi^{2} R^{2} \rho+d^{2} \rho^{2}}\right) / 6 \rho>\phi R,
\end{aligned}
$$

- Proof. The number of vehicles that connect one swath-corridor, which is at a distance of $i \cdot d$ from the city center, with its external area is $H \theta / 2 \pi$. The total demand generated in that swath and in its external area is $\Lambda\left[4 \phi^{2} R^{2}-\left(4 i^{2}-\right.\right.$ $\left.4 i+1) \rho d^{2}\right] / 4 \phi^{2} R^{2}$, and the probability that this demand has its destination in the swath is $i d^{2} / \phi^{2} R^{2}$. Mutiplying these three terms, the number of passengers carried by one vehicle is $H \Lambda \theta i d^{2}\left[4 \phi^{2} R^{2}-\left(4 i^{2}-4 i+1\right) \rho d^{2}\right] / 8 \pi \phi^{4} R^{4}$. The most occupied vehicles are those that serve the swath at a distance of $\left(2 d \rho+\sqrt{12 \phi^{2} R^{2} \rho+d^{2} \rho^{2}}\right) / 6 \rho$ from the city center. When this distance is 
inside the central attractant area, the occupancy is $O_{i}=H \Lambda \theta d\left[d \rho\left(36 \phi^{2} R^{2}\right.\right.$ $\left.\left.-\rho d^{2}\right)+\rho^{1 / 2}\left(12 \phi^{2} R^{2}+\rho d^{2}\right)^{3 / 2}\right] / 216 \pi \phi^{4} R^{4} \rho$. Otherwise, the occupancy is a monotonically increasing function in the central attractant area, and therefore the swath with the maximum occupancy is at the boundary of this area since there are no swath-corridors in the periphery. Then, $O_{e}=$ $H \Lambda \theta d(2 \phi R-d)\left[\phi^{2} R^{2}(1-\rho)+\rho d(2 \phi R-d)\right] / 4 \pi \phi^{4} R^{4}$ is the occupancy in the most external swath-corridor. Finally, as in all cases, a safety factor $(S F)$ is included.

\section{Appendix 2. Alternative Criterion on Route Choice in Direct-Trip-Based and Transfer-Based Structures}

To get a simple and compact formulation, we assume that some criteria on route choice prevail over the others. Two criteria are compared: the shortest-distance path and the path with the minimum number of transfers. This last criterion is equivalent to direct trips in a direct-trip-based structure since all of origin-destination pairs can be completed with no transfers. In this appendix, we demonstrate that the criterion chosen as prevalent in Section 2.4.2 is the alternative that provides the shortest travel times. For reasons of space, the formulation for the case that the prevailing criterion is different from Section 2.4.2 is skipped.

In the direct-trip-based structure, we assume that a direct trip is more competitive than the alternative shortest-distance path through the city center where users have to make a transfer. The difference in total travel time between the shortestdistance path and the direct trip is $(2+\theta / 2-\varphi) r / v_{c}+w_{W} H r / s+w_{t} \delta / w-w_{A}(d-s) /$ $4 w$, being $\varphi$ the angle between the origin and destination and $r$ the distance from the city center to the most central end of the trip between origin and destination. This result depends on the commercial and pedestrian speeds, service headway, transfer penalty, corridor and stop spacings, user time perceptions, and origin and destination locations. Focusing on the base case study, this difference is always positive for all users; that is, the direct trip is always the best choice. For an unconstrained headway scenario, Fig. 11a shows the total cost curve for different values of $\phi$ considering the alternative route choice where the shortest-distance criterion prevails. For any degree of dispersion, the total cost assuming this option (dashed black line) is higher than in the base scenario of Section 3 (dashed grey line). As a result, the range of values of $\phi$ where the direct-trip-based structure is the cheapest alternative is shorter, $\phi_{R a \rightarrow D T}$ is greater and $\phi_{D T \rightarrow H y}$ is smaller. Analyzing other scenarios of demand, city size, transfer penalty and unit costs such as in Section 3.2, the results are similar to the base case study. Following the shortest-distance criterion, the value of $\phi$ from which a hybrid scheme is a better solution than a direct-trip-based structure is smaller than following the criterion of minimum number of transfers. At the same time, this value is higher when the comparison is between radial and direct-trip-based networks (Fig. 11b). Therefore, 
the applicability of the direct-trip-based structure is reduced. For that reason, the model works with the criterion of minimum number of transfers.
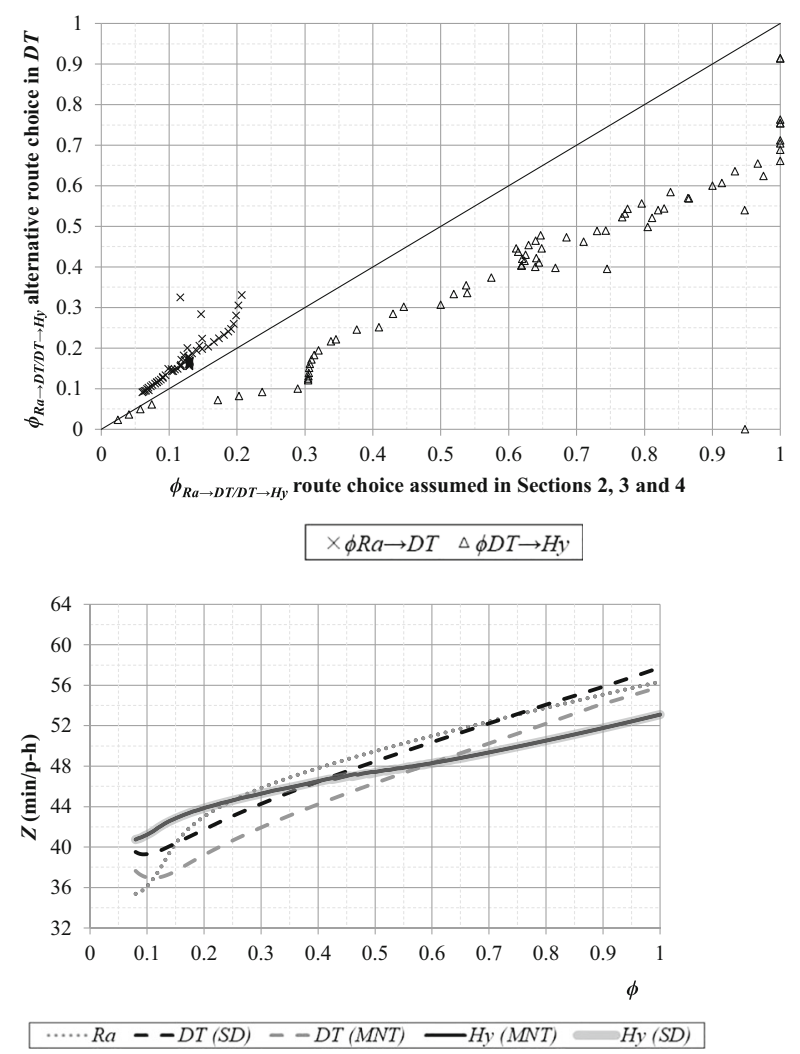

Fig. 11 Comparison between criteria on route choice in direct-trip-based and hybrid structures

On the other hand, if the hybrid network is optimized considering that the criterion of minimum number of transfers prevails, the results are the same as in Section 3. The decision variable $\alpha$ is always coincident with the degree of dispersion $\phi$. Therefore, there are no discrepancies between alternative route choices. To connect any origindestination pair, there is a route that meets both criteria. For that reason, the curves of total system cost are coincident (solid black and grey lines in Fig. 11a).

\section{Appendix 3: Sensitivity Analysis of the Transit Network Design Model}

The heterogeneity of urban areas could impede the transit system from adopting the optimal network configuration. As a consequence, the total system cost increases and the applicability of each network structure could vary. To evaluate possible effects, we make a sensitivity analysis about the variation of the total system cost with regard to changes in the decision variables. In this way, we can evaluate the robustness of the analytical model around the optimal network configuration. Figure 12 exhibits in the vertical axis the ratio between total cost when one decision variable is modified $Z$ ' and 
total cost for the optimal configuration $Z^{*}$, and in the horizontal axis, the ratio of variation of the decision variable under analysis $\left(s^{\prime} / s^{*}, \theta^{\prime} / \theta^{*}, H^{\prime} / H^{*}, \alpha^{\prime} / \alpha^{*}\right.$ or $\left.d^{\prime} / d^{*}\right)$. In each graph, only one decision variable is modified while the others are constant and equal to the optimum. We consider an unconstrained service headway scenario and the parameter $\phi$ equal to 1 ; therefore, $f_{d}$ is irrelevant in this case. For other values of these parameters, the evolution of the total cost is similar.

According to the results, the analytical model is robust for all the network structures. When the decision variables vary around $30 \%$, the total cost increases less than $2 \%$ in most of cases. For the direct-trip-based structure and the radial network, this increase is bounded to $3 \%$ when the characteristics of the city force the final network design to reduce the stop spacing. The greatest increase happens in the hybrid scheme when parameter $\alpha$ varies from 1 to 0.7 . In this case, the increase almost reaches $5 \%$. However, when the variation of the decision variables is around $15 \%$, the upper bound of the total cost growth is $1.5 \%$.

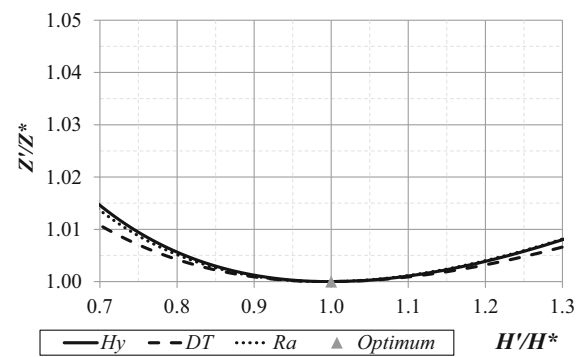

(a) Headway

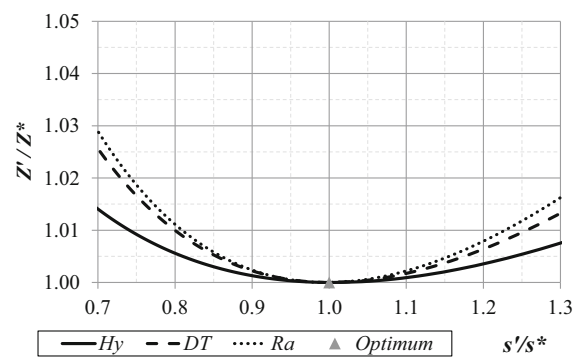

(c) Stop and line spacing

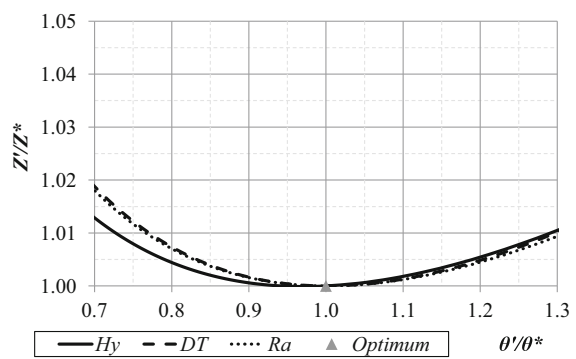

(b) Angular line spacing

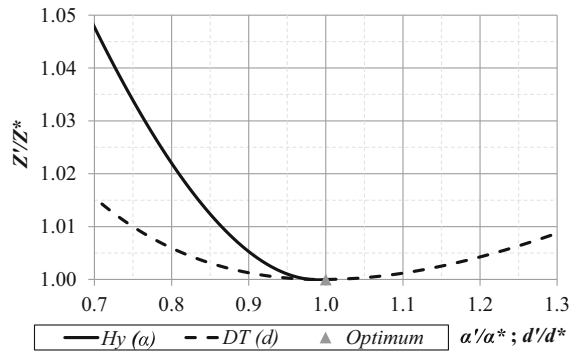

(d) Corridor spacing (DT) and decision variable $\alpha(\mathrm{Hy})$

Fig. 12 Robustness of the analytical model with regard to the total system cost around the optimal network configuration

However, the previous increases of the total cost would have limited effects on the applicability of each structure for different reasons: (i) great changes of the decision variables are required to produce significant increases in the total cost; (ii) the total cost growths obtained due to the variation of one decision variable could be reduced adjusting the other variables as design levers to compensate for those growths; (iii) the factor that determines the applicability of each structure is the difference between their respective total system costs, then the variation of that difference could be smaller than the variation of each particular total cost; and finally, (iv) if urban constraints forces an adjustment to the optimal value of one decision variable, Fig. 12 shows that it 
is always better to adopt higher values of that variable since the total cost increases with a smaller slope in that direction (in this case, it does not apply for $\alpha$ because this variable cannot be greater than 1).

Based on these reasons and the results in Fig. 12, we can assume that the main conclusions of this paper are not invalidated for possible adjustments to the optimal values of the decision variables. For further information, readers can consult Badia (2016) where a more exhaustive sensitivity analysis includes the effects of each decision variable on the different partial costs of the objective function (1) and other metrics of the bus network.

Open Access This article is distributed under the terms of the Creative Commons Attribution 4.0 International License (http://creativecommons.org/licenses/by/4.0/), which permits unrestricted use, distribution, and reproduction in any medium, provided you give appropriate credit to the original author(s) and the source, provide a link to the Creative Commons license, and indicate if changes were made.

\section{References}

Aguilera A (2005) Growth in commuting distances in French polycentric metropolitan areas: Paris, Lyon and Marseille. Urban Stud 42(9):1537-1547

Amirgholy M, Shahabi M, Gao HO (2017) Optimal design of sustainable transit systems in congested urban networks: A macroscopic approach. Transp Res Part E 103:261-285

Anas A, Arnott R, Small K (1998) Urban spatial structure. J Econ Lit 36(3):1426-1464

Baaj MH, Mahmassami HS (1995) Hybrid route generation heuristic algorithm for the design of transit networks. Transp Res Part C 3(1):31-50

Badia H (2016) Comparison of bus network structures versus urban dispersion: A monocentric analytical approach. Evidences from Barcelona's bus network. PhD Dissertation. Universitat Politècnica de Catalunya

Badia H, Argote-Cabanero J, Daganzo CF (2017) How network structure can boost and shape the demand for bus transit. Transp Res Part A 103:83-94

Badia H, Estrada M, Robusté F (2014) Competitive transit network design in cities with radial street patterns. Transp Res Part B 59:161-181

Badia H, Estrada M, Robusté F (2016) Bus network structure and mobility pattern: A monocentric analytical approach on a grid street layout. Transp Res Part B 93:37-56

Baumont C, Ertur C, Gallo J (2004) Spatial analysis of employment and population density: the case of the agglomeration of Dijon 1999. Geogr Anal 36(2):146-176

Bertaud A (2004) The spatial organization of cities: deliberate outcome or unforeseen consequence? IURD Working Paper Series WP-2004-01. Institute of Urban and Regional Development, Berkeley

Bontje M, Burdack J (2005) Edge cities, European-style: examples from Paris and the Randstad. Cities 22(4): $317-330$

Chen H, Gu W, Cassidy MJ, Daganzo CF (2015) Optimal transit service atop ring-radial and grid street networks: A continuum approximation design method and comparisons. Transp Res Part B 81:755774

Chen J, Liu Z, Wang S, Chen X (2018) Continuum approximation modeling of transit network design considering local route service and short-turn strategy. Transp Res Part E 119:165-188

Chen PW, Nie YM (2018) Optimal design of demand adaptive paired-line hybrid transit: Case of radial route structure. Transp Res Part E 110:71-89

Cheng H, Mao C, Madanat S, Horvath A (2018) Minimizing the total costs of urban transit systems can reduce greenhouse gas emissions: The case of San Francisco. Transp Policy 66:40-48

Craig SG, Ng PT (2001) Using quantile smoothing splines to identify employment subcenters in a multicentric urban area. J Urban Econ 49(1):100-120

Currie G, Loader C (2010) Bus network planning for transfers and the network effect in Melbourne, Australia. Transp Res Rec 2145:8-17

Daganzo CF (2010) Structure of competitive transit networks. Transp Res Part B 44(4):434-446 
Daganzo CF, Gayah VV, Gonzales EJ (2012) The potential of parsimonious models for understanding large scale transportation systems and answering big picture questions. EURO J Transp Logist 1(12):47-65

Dickinson RE (1961) The West European City. A Geographical Interpretation, 2nd edn. Routledge and Kegan Paul Ltd., London

Dodson J, Mees P, Stone J, Bruke M (2011) The principles of public transport network planning: a review of the emerging literature with select examples. Urban Res Program, Issues Paper 15

Estrada M, Roca-Riu M, Badia H, Robusté F, Daganzo CF (2011) Design and implementation of efficient transit networks: Procedure, case study and validity test. Transp Res Part A 45(9):935-950

Fan W, Mei Y, Gu W (2018) Optimal design of intersecting bimodal transit networks in a grid city. Transp Res Part B 111:203-226

Fielbaum A, Jara-Diaz S, Gschwender A (2016) Optimal public transport networks for general urban structure. Transp Res Part B 97:298-313

Fielbaum A, Jara-Diaz S, Gschwender A (2017) A parametric description of cities for the normative analysis of transport systems. Netw Spat Econ 17(2):343-365

Galster G, Hanson R, Ratcliffe MR, Wolman H, Coleman S, Freihage J (2001) Wrestling sprawl to the ground: defining and measuring an elusive concept. Hous Policy Debate 12(4):681-717

Giuliano G, Small KA (1991) Subcenters in the Los Angeles region. Reg Sci Urban Econ 21(2):163-182

Griffith DA, Wong DW (2007) Modeling population density across major US cities: a polycentric spatial regression approach. J Geogr Syst 9(1):53-75

Lee B (2007) "Edge" or "edgeless" cities? Urban spatial structure in US metropolitan areas, 1980 to 2000. J Reg Sci 47(3):479-515

Leurent F, Li S, Badia H (2019) Structural design of a hierarchical urban transit network integrating modal choice and environmental impacts. Transp, Res Procedia 37:99-106

Li ZC, Wang YD (2018) Analysis of multimodal two-dimensional urban system equilibrium for cordon toll pricing and bus service design. Transp Res Part B 111:244-265

Li ZC, Wang YD, Lam WH, Sumalee A, Choi K (2014) Design of sustainable cordon toll pricing schemes in a monocentric city. Netw Spat Econ 14(2):133-158

Lynch K (1962) Site Planning. MIT Press, Cambridge

Mees P (2000) A very public solution: transport in the dispersed city. Melbourne University Press, Melbourne

Moccia L, Giallombardo G, Laporte G (2017) Models for technology choice in a transit corridor with elastic demand. Transp Res Part B 104:733-756

Moccia L, Laporte G (2016) Improved models for technology choice in a transit corridor with fixed demand. Transp Res Part B 83:245-270

Newell GF (1979) Some issues relating to the optimal design of bus routes. Transp Sci 13(1):20-35

Nielsen G, Nelson JD, Mulley C, Tegner G, Lind G, Lange T (2005) Public transport - Planning the networks. HiTrans Best Practice Guide 2, Stavanger

Pereira RHM, Nadalin V, Monasterio L, Albuquerque PHM (2013) Urban centrality: a simple index. Geogr Anal 45(1):77-89

Rahimi M, Amirgholy M, Gonzales EJ (2018) System modeling of demand responsive transportation services: Evaluating cost efficiency of service and coordinated taxi usage. Transp Res Part E 112:66-83

Riguelle F, Thomas I, Verhetsel A (2007) Measuring urban polycentrism: a European case study and its implications. J Econ Geogr 7:193-215

Rodrigue JP, Comtois C, Slack B (2006) The geography of transport systems. Routledge, London

Saidi S, Wirasinghe SC, Kattan L, Esmaeilnejad S (2017) A generalized framework for complex urban rail transit network analysis. Transpor A: Transp Sci 13(10):874-892

Smeed RJ (1965) A theoretical model of commuter traffic in towns. IMA J Appl Math 1(3):208-225

Sun H, Forsythe W, Waters N (2007) Modeling urban land use change and urban sprawl: Calgary, Alberta, Canada. Netw Spat Econ 7(4):353-376

Tan T (1966) Road networks in an expanding circular city. Oper Res 14(4):607-613

Thompson GL (1977) Planning considerations for alternative transit route structures. J Am Inst Plann 43(2): 158-168

Tirachini A, Hensher DA, Jara-Díaz SR (2010) Comparing operator and users costs of light rail, heavy rail and bus rapid transit over a radial public transport network. Res Transp Econ 29(1):231-242

TRB (Transportation Research Board) (2003) Transit Capacity and Quality of Service Manual (TCQSM) (2nd ed.). In: Kittelson \& Associates, Inc., KFH Group, Inc., Parsons Brinckerhoff Quade \& Douglass, Inc., Hunter-Zaworski K (eds) Transportation Research Board. National Academy Press, Washington, DC

Tsekeris T, Geroliminis N (2013) City size, network structure and traffic congestion. J Urban Econ 76:1-14 
Van Nes R (2002) Design of multimodal transport networks, a hierarchical approach. TRAIL Thesis Series T2002/5, DUP, Delft University, the Netherland

Vaughan R (1986) Optimum polar networks for an urban bus system with a many-to-many travel demand. Transp Res Part B 20(3):215-224

Vuchic V (2005) Urban Transit: Operations, Planning and Economics. John Wiley and Sons, Hoboken

Zhao F (2006) Large-scale transit network optimization by minimizing user cost and transfers. J Public Transp 9(2):107-129

Publisher's Note Springer Nature remains neutral with regard to jurisdictional claims in published maps and institutional affiliations. 